\title{
PALEOBIOGEOGRAFÍA Y PALEOGEOGRAFÍA DEL MAASTRICHTIENSE MARINO DE LA PATAGONIA, TIERRA DEL FUEGO Y LA PLATAFORMA CONTINENTAL ARGENTINA, SEGÚN SUS FORAMINÍFEROS BENTÓNICOS
}

\author{
Carolina NÁNEEZ y Norberto MALUMIÁN² \\ ${ }^{1,2}$ Servicio Geológico Minero Argentino y CONICET, Benjamín Lavaissé 1194, \\ (C1107BJD) Buenos Aires, Argentina. \\ ${ }^{1}$ carolina.nanez@yahoo.com.²n.malumian@yahoo.com
}

Náñez, C. \& Malumián, N. 2008. Paleobiogeografía y paleogeografía del Maastrichtiense marino de la Patagonia, Tierra del Fuego y la Plataforma Continental Argentina, según sus foraminíferos bentónicos. [The paleobiogeography and paleogeography of marine Maastrichtian of Patagonia, Tierra del Fuego and the Argentinean Continental Shelf, based on benthic foraminifera.] Revista Española de Paleontología, 23 (2), 273-300. ISSN 0213-6937.

\begin{abstract}
The first Atlantic transgression that flooded Patagonia was Maastrichtian in age and persisted up to the Danian. It surpassed in extension the following Cenozoic transgressions, but there are no solid evidences of a trans South American paleosea-way connecting the Austral and Peruvian coast and/or Caribbean seas during the Maastrichtian. The extra Andean Patagonian area and the wide Argentinean Continental Platform gave place to a shallow sea, with extended marginal environments reflected by several low diversity foraminiferal assemblages. These assemblages provided us the geographic context to construct a reliable distribution map of marine Maastrichtian sediments. In the Austral or Magallanes Basin, a retroarc basin with clastic sedimentation, three foraminiferal assemblages of extreme environments are recognized: one of typical cosmopolitan agglutinated foraminifera from turbidite settings of the Fuegian Andes; a second agglutinated assemblage of very low diversity from marginal environments of the stable platform; and a mostly calcareous assemblage from platform environments, which has affinities with the Upper Cretaceous of New Zealand and Southern Australia, of similar paleolatitudes at that time. In the adjacent offshore Malvinas Basin, the Maastrichtian is scarcely represented, although emblematic Austral species such as "Frondicularia" rakauroana are recorded. Conversely, Northern Patagonia contains conspicuous endemic taxa recorded in the Golfo San Jorge Basin, of intracratonic position, and especially in the Neuquina-on shore Colorado Basin, a foreland basin under a semi-arid climate with mixed clastic-carbonate biogenic deposits. Seven low diversity assemblages are recognized in the Maastrichtian of northern Patagonia, mostly dominated by calcareous foraminifers: (1) in the Roca Formation, northernmost Neuquina Basin, a poor assemblage of miliolids, Protelphidium primitivum n. sp. and Tehuelchella caminosi n. gen. n. sp.; (2) at the base of the Jagüel Formation in the Bajo de Añelo area, dominated by glandulinids, discorbids and frequent polymorphinids; (3) in the Jagüel Formation, at the Liu Malal locality, an assemblage dominated by trochospiral forms, and characterised by the presence of Rosalina patagonica n. sp., and Protelphidium primitivum n. sp.; (4) in the Aguada Cecilio Member of the Arroyo Barbudo Formation, an almost monospecific assemblage of Tehuelchella caminosi n. gen. n. sp; (5) in the La Colina Formation, Bajada Moreno locality, a very low diversity assemblage comprising up to $10 \%$ of monolocular calcareous foraminifera, mainly characterised by Favolagena ardolinoi; (6) the most diversified assemblage, which is dominated by Buliminida, comprises Hiltermannella kochi and a number of endemic taxa, recorded from dark mudstones with scarce megafauna, related to dysaerobic environments of the Jagüel Formation. Only one very low diversity assemblage of agglutinated foraminifera of very restricted distribution is found: (7) in the Lefipán Formation, an almost monospecific assemblage of $\mathrm{Ha}$ plophragmoides sp., related to deltaic paleoenvironments. An assemblage of Austral character was found in the off shore Colorado Basin, on the Argentinean Continental Platform, which comprises Notoplanulina rakauroana, Coryphostoma incrassata gigantea, Stensioeina beccariiformis, Praebulimina carseyae, Allomorphina cretacea, Quadrimorphina camerata, Alabamina creta; one third of the specimens are agglutinated forms, including: Reticulophragmium sp., Rzehakina fissistomata, Trochammina sp. and Dorothia bulletta. The new taxa proposed in this article are apparently restricted to the Maastrichtian, and as most of the Maastrichtian endemic forms, they were not found in the well known Patagonian Danian, which carries a Midway type assemblage with few endemic taxa. Including the new taxa, there are 95 species recorded (85 calcareous, and 10 agglutinated) in the
\end{abstract}


Maastrichtian of the Northern Patagonia region, ca. $15 \%$ of them endemic to the Southern Hemisphere, and $10 \%$ endemic to the region. As a whole in South America the assemblages of Maastrichtian foraminifera from Patagonia differ greatly from those of the paleo-West Indian Province and could be considered belonging to the Austral realm, which includes the Northern Patagonia as an endemic center within an inner sea. The records of Lagenoglandulina, Hiltermannella, Rosalina, Protelphidium and Tehuelchella in the Patagonian Maastrichtian are the oldest known for these genera, suggesting their southern extratropical origin; they characterize the Austral assemblages, whereas Tehuelchella, morphologically similar to Buccella, would represent the origin of the Buccella kingdom of the modern Patagonian shelf.

Keywords: Foraminifera, Maastrichtian, Patagonia, paleobiogeography, paleogeography, new genus and new species.

\section{RESUMEN}

La primera transgresión atlántica reconocida en la Patagonia es de edad Maastrichtiense, y superó en extensión a las posteriores transgresiones cenozoicas. La Patagonia extraandina y la extensa Plataforma Continental Argentina adyacente dieron lugar a un mar somero, con ambientes muy marginales ampliamente distribuidos y reflejados en sus asociaciones de foraminíferos de muy baja diversidad y de gran significado paleobiogeográfico. A partir de estas asociaciones, se han confeccionado dos mapas: el de la distribución de las sedimentitas marinas maastrichtienses, y el paleogeográfico correspondiente. El mar permaneció hasta el Daniense, sin que se compruebe una comunicación mediante un brazo marino intracontinental con la costa peruana y/o el Caribe. En la cuenca Austral, de neta sedimentación clástica, se reconocen tres asociaciones de foraminíferos de ambientes extremos: una, de foraminíferos aglutinados cosmopolitas en ambientes turbidíticos de los Andes Fueguinos; una segunda asociación de foraminíferos aglutinados de muy baja diversidad de ambientes marginales de plataforma estable; y una tercera, dominada por foraminíferos calcáreos de plataforma, afines a los del Cretácico Superior de Nueva Zelanda y del sur de Australia, que a la sazón compartían paleolatitudes similares. En contraste, las asociaciones de la cuenca del Golfo de San Jorge, y en especial de la cuenca Neuquina-Colorado costa adentro, con depósitos mixtos biogénicos calcáreos-clásticos, contienen conspicuos géneros y especies endémicos, a los que aquí se agregan: Tehuelchella caminosi n. gen. n. sp., Rosalina patagonica n. sp. y Protelphidium primitivum n. sp. Estas nuevas especies, como la mayoría de las endémicas maastrichtienses, no se han hallado en el bien conocido Daniense patagónico. Incluyendo los nuevos taxones propuestos, en el Maastrichtiense de la Patagonia septentrional se han reconocido en total 95 especies (85 de calcáreos y 10 de aglutinados), de las cuales aproximadamente $15 \%$ son aparentemente endémicas del Hemisferio Sur, y el 10\% endémicas de la región. El conjunto de las asociaciones del Maastrichtiense de la Patagonia difiere netamente de aquellas de la paleo-Provincia de las Indias Occidentales, y puede ser considerado perteneciente al reino Austral, incluyendo Patagonia septentrional como un centro endémico desarrollado en un somero mar marginal. Los registros de Lagenoglandulina, Rosalina, Hiltermannella, Protelphidium y Tehuelchella en el Maastrichtiense patagónico son los más antiguos conocidos para estos géneros, sugiriendo su origen austral y extratropical. Estos géneros caracterizan las asociaciones australes, y en particular Tehuelchella, morfológicamente similar a Buccella, representaría el origen del reino de Buccella de la actual plataforma patagónica.

\section{Palabras clave: Paleogeografía, Paleobiogeografía, Foraminifera, Maastrichtiense, Patagonia, nuevo gé- nero, nuevas especies.}

\section{INTRODUCCIÓN}

La primera transgresión atlántica reconocida en la tierra emergida en el sur de América del Sur, se produce en el Maastrichtiense y da lugar a un mar que se retira a fines del Daniense. Esta transgresión cubre gran parte de la Patagonia extrandina y la región fueguina (Fig. 1). Si bien supera en extensión a las siguientes transgresiones cenozoicas, no alcanza gran profundidad y da lugar a diversos ambientes someros de plataforma. Mediante sus foraminíferos, se reconoce en todas las cuencas patagónicas, excepto en la de Península de Valdés.
Varios autores han tratado de establecer la distribución geográfica de las sedimentitas maastrichtienses marinas de la Patagonia (Camacho, 1967; Cecioni, 1970; Malumián et al., 1983; Riccardi, 1987). Incluso se ha bosquejado la paleogeografía sudamericana dentro de panoramas mundiales (Barron, 1987; Huber, 1992 a,b; Markwick \& Valdés, 2004), con modelos que se caracterizan por indicar un brazo de mar transcontinental, que internamente conecta las regiones australes con la peruana y/o la caribeña, basados en interpretaciones generalizadas para el Cretácico Superior (Uliana \& Biddle, 1988; Macellari, 1988; Salfity \& Zambrano, 1990). El control estrati- 
gráfico y paleobiogeográfico del Maastrichtiense en las cuencas patagónicas se obtuvo mayormente en la última década, principalmente mediante el reconocimiento de asociaciones de foraminíferos con definidas características paleoecológicas. En este artículo, se describen nuevos taxones dominantes en paleoambientes muy someros, que son biogeográficamente significativos, ya que los taxones de aguas profundas son generalmente cosmopolitas. A partir de estas asociaciones de aguas someras se trazan los límites de la distribución de las sedimentitas marinas maastrichtienses, y se discute el significado biogeográfico con el objetivo de indagar sobre los orígenes de los foraminíferos cenozoicos de la Patagonia y la plataforma continental adyacente.

Los autores han evaluado los foraminíferos de todas las entidades estratigráficas asignadas al Maastrichtiense distribuidas en las distintas cuencas patagónicas y fueguinas (Fig. 1). Asimismo, han revisado aquellas consideradas de edad maastrichtiense en estudios paleogeográficos previos y que resultaron ser eocenas, como la Formación Man Aike, en la Patagonia, y la Formación Leticia, en la Isla de Tierra del Fuego; o anteriormente consideradas de mayor antigüedad, y que son maastrichtienses, como la Formación Policarpo. Por lo tanto, es la primera vez que se proyecta un mapa paleogeográfico del Maastrichtiense marino controlado en todas las cuencas patagónicas y de la plataforma continental adyacente.

Todo intento de análisis biogeográfico tropieza con la subjetividad de la taxonomía: se ha sugerido que un mismo taxón ha recibido un nombre diferente en cada continente (Bettenstaedt \& Wicher, 1955); en contraste, los estudios moleculares recientes están revelando que especies diferentes y que habitan áreas geográficas distintas pueden poseer caparazones muy similares e indistintos (ej. DeVargas et al., 1999). Por otro lado, el grado de endemismo aparente es función del conocimiento de la distribución de los taxones: un taxón es endémico mientras no se lo identifique en otra región; debe tenerse en cuenta que las ausencias son datos negativos per se y por ende no verificables.

\section{ANTECEDENTES PALEOBIOGEOGRÁFICOS- OCEANOGRÁFICOS}

En muy grandes rasgos, dentro de América del Sur, las asociaciones de foraminíferos bentónicos del Cretácico de la Patagonia y de la Isla de Tierra del Fuego, se han incluido en las faunas australes y diferenciado marcadamente de aquellas de las regiones tropicales por características negativas, dadas por la baja diversidad y la ausencia de taxones tropicales, particularmente macroforaminíferos (Scheibnerová, 1971; Malumián \& Masiuk, 1973; Sliter, 1977).
La Provincia biogeográfica de las Indias Occidentales, también denominada Antillana o Caribeña, ya reconocida por Tinoco (1975), fue elevada al rango de reino, denominado Noratlántico Central-Tetis Occidental por Koutsoukos \& Klasz (2000); contiene los géneros Siphogenerinoides Cushman, 1927 y Orthokarstenia Dietrich, 1935 que caracterizan al Cretácico Superior desde Venezuela hasta Perú. En Brasil, se ilustraron varias especies para la Formación Gramame, Maastrichtiense de Pernambuco (Tinoco, 1978), la latitud más alta (aprox. $10^{\circ} \mathrm{LS}$ ) en que se han encontrado estos géneros por la costa atlántica. De esta manera, se distinguen para el Maastrichtiense dos entidades biogeográficas mayores dentro de América del Sur: una Septentrional y otra Austral.

El límite entre ambas entidades, en la vertiente pacífica, se situaría dentro de una zona indefinida muy amplia, debido a la falta de sedimentitas maastrichtienses marinas desde el sur del Perú hasta el norte de Chile inclusive, pues afloran aproximadamente a $36^{\circ} \mathrm{LS}$ en Chile central. Allí, las clásicas capas de la Isla de Quiriquina, asignadas al Maastrichtiense por moluscos considerados de una provincia de transición (ver más adelante), podrían indicar una conexión atlántica-pacífica, pero al desconocerse su contenido microfaunístico no puede aventurarse la existencia de tal conexión. Otra zona indefinida de similar extensión se sitúa en la vertiente atlántica entre Pernambuco (norte del Brasil) y la cuenca del Salado inclusive (Argentina central), pero aquí el contexto es más problemático, pues las microfaunas maastrichtienses conocidas en Brasil son de aguas profundas, de menor relevancia biogeográfica, y de difícil comparación con las asociaciones de aguas someras conocidas y dominantes en el Cono Sur.

La conexión mediante un brazo transcontinental entre las regiones Austral y la peruana y/o caribeña (Macellari, 1988; Markwick \& Valdes, 2004) carece de fundamentos micropaleontológicos, y la reciente asignación al Mioceno de formaciones marinas argentinas (Marengo et al., 2005) previamente consideradas maastrichtienses, y extendidas en el interior del país a través de la cuenca del Salado, le restan sustento estratigráfico a la existencia de tal brazo.

La apreciación del carácter austral de las faunas patagónicas se remonta a los primeros estudios de invertebrados de fines del siglo XIX, y al comienzo del estudio sistemático de los foraminíferos cretácicos en la Argentina (Malumián \& Masiuk, 1973), que notaron los vínculos con Nueva Zelanda, Australia y Antártida. Entre las primeras referencias, los neocelandeses reconocieron los elementos paleoaustrales integrantes de un centro o región endémico (Fleming, 1962; Stevens, 1980), que Kauffman (1973), para el Maastrichtiense, dividió en dos subprovincias; Scheibnerová (1971, 1973), Sliter (1977), Zinsmeister (1979), Krasheninnikov \& Basov (1986), lo elevaron a nivel de provincia, y Huber (1992a,b; 1994) lo consideró un reino. En el extremo austral de América del Sur, 
se reconoce la misma situación señalada para Australasia por McGowran (1978): las asociaciones son provinciales, pero con "ventanas" provistas por excursiones desde los trópicos, que dan una idea de las variaciones climáticas (Malumián \& Náñez, 1996).

Los foraminíferos del Maastrichtiense-Daniense de la

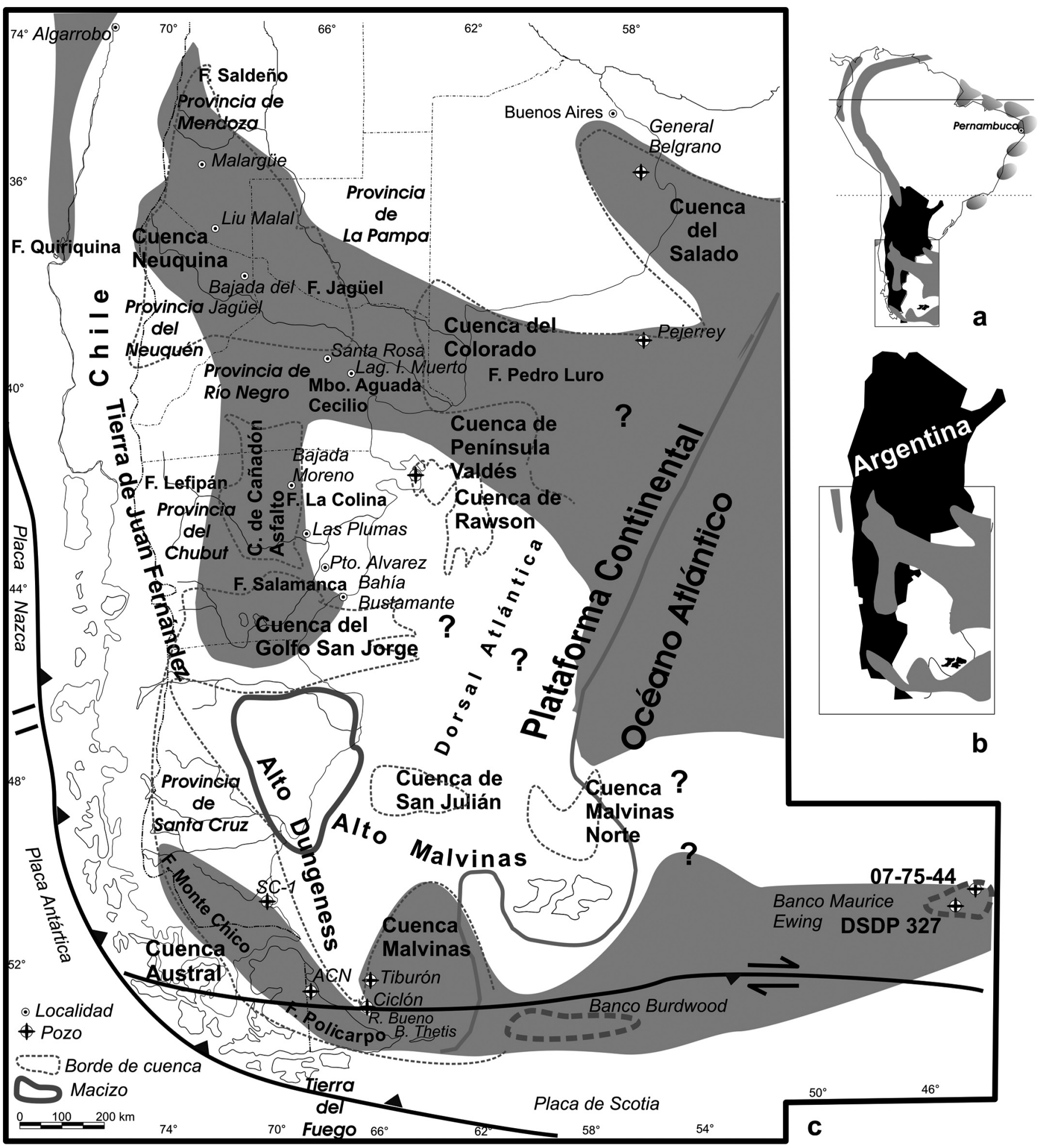

Figura 1. a, distribución esquemática del Maastrichtiense marino y situación relativa de la Patagonia y Tierra del Fuego dentro de América del Sur. b, situación relativa del área de estudio en la Argentina. c, distribución de las sedimentitas marinas maastrichtienses (en gris), localidades, cuencas y formaciones mencionadas en el texto.

$a$, Relative position of the Patagonia and the Tierra del Fuego island and schematic distribution of the marine Maastrichtian in South America. $\boldsymbol{b}$, relative position of the study area in Argentina. $\boldsymbol{c}$, distribution of the Maastrichtian marine sediments (grey areas), localities, basins, and formations mentioned in the text. 
isla Seymour, península Antártica, pertenecen a la provincia Austral (Huber, 1988). Basado en foraminíferos planctónicos, Huber (1992b) incluye las latitudes de la Patagonia septentrional en una zona de transición entre los reinos del Tetis y el Austral, y puntualiza que los gradientes latitudinales de diversidad fueron más débiles durante el Campaniense temprano y más pronunciados durante el tardío y el Maastrichtiense. La máxima diversidad de taxones australes provinciales se produce durante el Campaniense tardío en los foraminíferos planctónicos y durante el Maastrichtiense temprano en el nanoplancton calcáreo (Huber \& Watkins, 1992). En el Maastrichtiense tardío, los foraminíferos planctónicos permiten reconocer un reino Austral, con especies endémicas polares que pueden llegar a formar el $60 \%$ de la fauna, y cuyas aguas enfrentaban la cuenca Austral. Asimismo, se reconoce un reino Transicional, cuyas aguas enfrentarían las cuencas de la Patagonia septentrional, y que no contiene elementos particulares, distinguiéndose del reino del Tetis por la diferente frecuencia de las especies (Nederbragt, 1998).

En el Maastrichtiense tardío, para ambientes profundos del Océano Atlántico Sur, se reconocieron cinco asociaciones de foraminíferos bentónicos, entre ellas la Asociación de Aguas Someras de Altas Latitudes (Widmark, 2002), que incluye varias especies comunes con la cuenca Austral.

El rasgo biogeográfico más sobresaliente de los foraminíferos del Cretácico Superior de la Argentina, en particular para el Maastrichtiense, es la disimilitud entre las asociaciones de la Patagonia septentrional y las de la cuenca Austral (Malumián \& Báez, 1976). La cuenca Austral tiene especies comunes con Nueva Zelanda y Australia (Malumián \& Masiuk, 1973), no reconocidas en la Patagonia septentrional. Malumián \& Báez (1976) explicaron la disimilitud mediante la idea de Groeber $(1959,1963)$ que propone una extendida barrera entre la cuenca Austral y la Patagonia septentrional, denominada Tierra de Juan Fernández, que se continúa por los altos de Dungeness y de Malvinas. Malumián (1979) resaltó esta disimilitud y la vinculó con la reconocida en el Océano Atlántico por Ciesielski et al. (1977) y Ciesielski \& Wise (1977), entre muestras del Cretácico Superior del fondo oceánico separadas latitudinalmente sólo por $62 \mathrm{Km}$, entre testigos del DSDP y Orcadas 07-75-44 en el banco Maurice Ewing (Fig. 1). Estos autores explicaron las notables diferencias reconocidas en las asociaciones de foraminíferos y nanoplancton calcáreo, por la convergencia de una corriente cálida de giro antihorario proveniente del norte y una fría del sur de giro horario sobre la plataforma o alto de Malvinas (Fig. 2), estructura que pudo actuar como barrera topográfica.

Bertels $(1979,1988)$ reconoció la disimilitud en las asociaciones, distinguiendo la Subprovincia Neuquén, Mendoza más cuenca del Colorado, diferenciada netamente de la cuenca Austral. Puntualizó que si bien no hay especies comunes entre la Patagonia septentrional y el África occidental, se encuentran afinidades entre algunos foraminíferos como Hiltermannella Bertels, 1971, y Gabonella de Klasz, Maire \& Meijer, 1960 (el último nombre por estar preocupado actualmente es substituido por Gabonita Dieni, 1974), y que las afinidades se manifiestan particularmente entre los ostrácodos, que poseen una mayor significación biogeográfica.

Una crítica común a todos estos intentos de dilucidar las relaciones biogeográficas entre los foraminíferos maastrichtienses de la Patagonia septentrional y de la cuenca Austral, es que no se comparaban asociaciones coevas (Malumián, 1990), pues los de la última cuenca, dada su pobre preservación o ausencia en vastas áreas del subsuelo, no fueron ilustrados hasta años recientes (Malumián \& Náñez, 2002 a,b).

Otros grupos fósiles contribuyen a los análisis paleobiogeográficos y paleoclimáticos. Bertels (1974, 1975, 1995) y Tambareau (1982) señalaron la afinidad de los ostrácodos maastrichtienses de Patagonia septentrional con los del África occidental, mientras que Dingle (1988) los incluye en su fauna pan-gondwánica D (PGFD) del Campaniense y Maastrichtiense, originada en el Cenomaniense, y migrante hacia el templado Atlántico Sur en momentos de nivel de mar alto.

Los nanofósiles calcáreos maastrichtienses de Patagonia septentrional son típicos de altas latitudes, y corresponderían a una expansión hacia el norte de la Bioprovincia del Plateau de Malvinas, que se extendería a latitudes de hasta $36^{\circ} \mathrm{S}$ (Concheyro \& Villa, 1996).

Mediante carofitas, Mussachio (2000) diferencia para el Cretácico Superior las provincias Atlántica y Andina Sudamericanas. La primera se distribuye en una faja costera desde la cuenca de Campos, en Brasil, hasta el sur de Mendoza, y exhibe un intercambio de ostrácodos y carofitas con África y Europa del Sur. La segunda, en cuencas pericratónicas interiores, muestra una fuerte vinculación con América Central, y las Rocallosas, en América del Norte.

En general, las asociaciones palinológicas del Maastrichtiense marino de la Patagonia reflejan condiciones someras, con alto contenido de dinoflagelados peridinoideos y un importante aporte de elementos de origen continental; con débil afinidad entre los dinoflagelados de Patagonia septentrional y los de la Formación Calafate de la cuenca Austral (Guerstein et al., 2004). Se reconocen dos asociaciones netamente diferenciables: la asociación de Areoligera spp., en la cuenca Neuquina-Colorado y llamativamente también en la Formación Chorrillo Chico, Magallanes; y la asociación de Manumiella spp., con dominancia de especies peridinoides, que se encuentran en la Cordillera Fueguina y en la península Antártica (Palamarczuk et al., 1998; Palamarczuk \& Olivero, 2000), planteando una divisoria biogeográfica dentro de la misma cuenca. 
La palinoflora de helechos y angiospermas de la sección inferior de la Formación Lefipán reflejaría una tierra arbustiva con áreas boscosas en condiciones templadas cálidas a posiblemente subtropicales. Si bien contiene taxones comunes con asociaciones australes, en sus aspectos generales difiere de las floras boscosas templadas de coníferas de altas latitudes (Baldoni \& Askin, 1993). Los más antiguos de granos de polen de Nothofagus, típicos de regiones australes, se citan para las formaciones Jagüel, Loncoche y Roca, en bajos porcentajes (Romero, 1973; Papú, 2002; Prámparo \& Papú, 2002, 2003).

Los gasterópodos maastrichtienses-paleocenos de cuenca Austral, sugieren una estrecha relación con faunas neocelandesas (Griffin \& Hünicken, 1994). En la Formación Lefipán, se diferenciaron dos asociaciones, mayormente de moluscos, una maastrichtiense, con formas afines y comunes, o características del Hemisferio Austral, en contraste con el endemismo de la daniense con débiles afinidades australes (Olivero et al., 1990; Medina \& Olivero, 1994). Los ammonites reflejan su endemicidad en la familia Kossmaticeratidae, cuya dominancia se restringe casi exclusivamente a la provincia austral (Macellari, 1985; Olivero \& Medina, 2000). En contraste, en la Patagonia septentrional, los ammonites se reducen a moldes internos de baculítidos (Riccardi et al., 1999), y escasos restos de gaudricerátidos (comunicación verbal de E. Olivero, 2007). Esta absoluta dominancia de baculítidos puede resultar de la mayor competitividad de los heteromorfos cuando las condiciones ambientales son inestables, o por un efecto tafonómico (Henderson \& McNamara, 1985).

En Patagonia septentrional, los equinoideos del Maastrichtiense-Daniense exhiben similitudes con componentes del Tetis, apoyando el patrón de intercambio propuesto por Néraudeau \& Mathey (2000), con la apertura del corredor a través del Sahara en el Cretácico Superior, mecanismo de dispersión que también se aplicaría a moluscos y decápodos. Durante el Maastrichtiense, en Patagonia septentrional dominaron moluscos con afinidades australes; para el Maastrichtiense tardío aparecieron los primeros bivalvos y decápodos derivados de bajas latitudes, que se hacen dominantes en el Daniense (Feldmann et al., 1995; Casadío, 1998; Casadío et al., 1999; Parma \& Casadío, 2005; Griffin et al., 2005; Feldmann \& Schweitzer, 2006). En los reptiles, al menos en Nueva Zelanda hay plesiosaurios pelágicos con características similares a los de la Patagonia septentrional (Gasparini et al., 2007).

La malacofauna de la Formación Quiriquina comparte muchas especies con la Patagonia, la península Antártica, Nueva Zelanda, Australia, India y África del Sur, con una declinación de la diversidad que comenzó mucho antes del final del Maastrichtiense (Stinnesbeck, 1986). Su rica fauna de peces, aunque marcadamente endémica, tiene semejanza con las contemporáneas de América del Norte (Suárez, 2001; Suárez \& Cappetta, 2004). En particular, una especie de Belonostomus Agassiz, 1834, común al Maastrichtiense de la Patagonia septentrional (Casamiquela, 1978), sugiere más afinidad con la fauna pacífica de América del Norte que con la atlántica de América del Sur (Brito \& Suárez, 2003). Los frecuentes dientes de tiburones y rayas indican aguas templado-cálidas al menos para la zona basal de la Formación Quiriquina (Muñoz \& Palma-Heldt, 2006)

En resumen, dentro de una variedad de consideraciones biogeográficas, existe consenso en reconocer una fauna y flora de carácter austral para el Maastrichtiense patagónico y fueguino; por una parte, con elementos provenientes de latitudes menores, manifiesta en ostrácodos, equinoideos y artrópodos; y por otra parte, de origen antártico y australiano por mamíferos que requieren de una conexión continental con la Antártida, o por lo menos insular, dado el selectivo intercambio de mamíferos entre la Patagonia y Australia (Pascual, 1998; Vizcaíno et al., 1998). Esta conexión aún no está esclarecida mediante datos paleomagnéticos, y no existe consenso sobre la evolución cinemática del Oroclino Patagónico, que incluye la Cordillera Fueguina, el banco Burdwood, las islas Orcadas y la península Antártica. Se han propuesto reconstrucciones que inicialmente se referían a un orógeno rectilíneo o con un arqueamiento original (Dalziel \& Elliot, 1971; Suárez \&

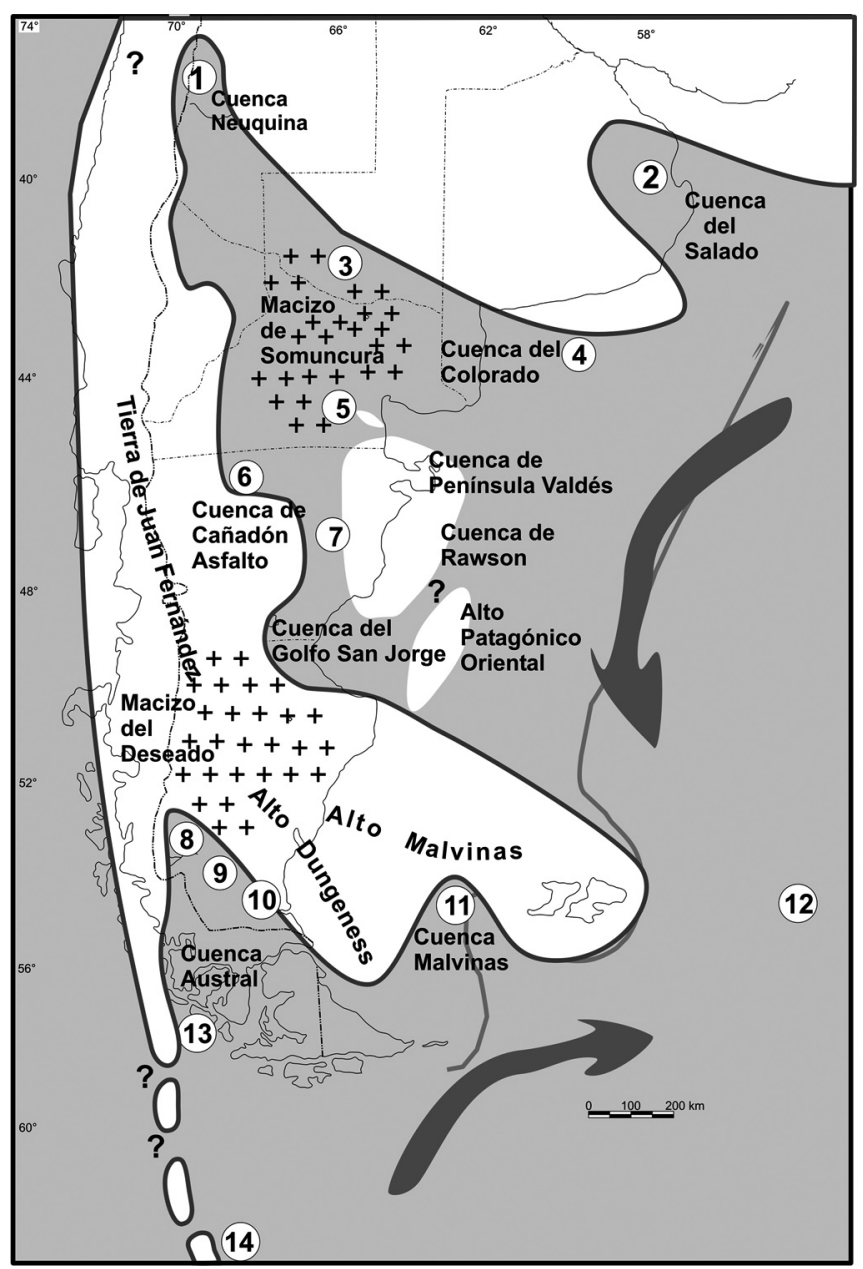


Pettigrew, 1976; Ramos, 1996), pero aún no existen datos paleomagnéticos suficientes para determinar si el arqueamiento del Oroclino Patagónico es primario o secundario (Rapalini, 2007). Las reconstrucciones consideran la existencia de un istmo que une Tierra del Fuego con la península Antártica (Barker et al., 1977).

\section{METODOLOGÍA}

Gran parte del material fue coleccionado durante años de estudios micropaleontológicos en apoyo al relevamiento de la carta geológica, habiendo intentado recuperar foraminíferos de todas las entidades estratigráficas de supuesta edad maastrichtiense de la Patagonia. Todos los conjuntos de foraminíferos fueron estudiados o directamente observados por los autores. Las ex- tendidas condiciones adversas para la vida de los foraminíferos planctónicos en las entidades estratigráficas investigadas resultan en una baja resolución bioestratigráfica, considerándose un Maastrichtiense no diferenciado a los fines de la confección del mapa de distribución de las sedimentitas maastrichtienses marinas. Los foraminíferos se han obtenido por desagregación y lavado de las muestras a través de tamiz de malla de $63 \mu \mathrm{m}$ de abertura y separación de los microfósiles con pincel. Las condiciones paleoambientales se estimaron expeditivamente en asociaciones de buena preservación sobre 300 ejemplares, considerando la composición taxonómica, relación planctónicos a bentónicos, el tamaño de la conchilla planctónica de mayor diámetro de la muestra (Murray, 1976), la diversidad y dominancia de morfotipos infaunales $v s$. epifaunales.

Los antecedentes de otros grupos fósiles se han detallado a fin de informar sobre la megafauna y palinología reconocida conjuntamente con las asociaciones de foraminíferos bentónicos descriptas, para su comparación en los aspectos paleobiogeográ-

Figura 2. Paleogeografia de la transgresión Maastrichtiense en la Patagonia y Tierra del Fuego y distribución de las asociaciones de foraminíferos más características en localidades claves. La paleocirculación según Ciesielski et al. (1977). La distribución de las áreas terrestres figurando un conjunto de islas entre Tierra del Fuego y la península Antártica es conjetural; alternativamente pudo existir un istmo (Barker et al., 1977). 1, Asociaciones más septentrionales formadas de miliólidos simples o con Protelphidium primitivum n. sp., en facies muy someras, calcáreas y oolíticas de las Formaciones Saldeño y Roca, cuenca Neuquina. 2, Asociaciones muy pobres, conchas muy pequeñas, y foraminíferos planctónicos solo representados por especies oportunistas del género Heterohelix Ehrenberg, 1843, sugiriendo proximidad de la costa, cuenca del Salado. 3, Asociaciones con bulimínidos dominantes, facies de fangolitas oscuras, disóxicas, con los ambientes de mayor profundidad en la Patagonia septentrional, Formación Jagüel, cuenca Neuquina-Colorado costa adentro. 4, Asociación de plataforma media con Notoplanulina rakauroana, Formación Pedro Luro, cuenca del Colorado costa afuera. 5, Asociación cuasi monoespecífica de Tehuelchella caminosi n. gen. n. sp., área de conexión entre las cuencas del Colorado y del Golfo de San Jorge, miembro Aguada Cecilio de la Formación Arroyo Barbudo. 6, Asociación de baja diversidad de foraminíferos aglutinados, en ambientes deltaicos hiposalinos, Formación Lefipán, depocentro intracratónico Lefipán-Paso del Sapo, cuenca de Cañadón Asfalto. 7, Pobres asociaciones de foraminíferos, Formación Salamanca, cuenca del Golfo de San Jorge. 8, Asociaciones de foraminíferos aglutinados de muy baja diversidad, paleoambientes hiposalinos, Formación Calafate. 9, Asociación de Coryphostoma incrassata gigantea, de plataforma media, en la glauconítica Formación Monte Chico, cuenca Austral. 10, Asociaciones de foraminíferos aglutinados, y calcáreos de baja diversidad, ambientes hiposalinos dominantes, margen estable de la cuenca Austral. 11, Asociación residual con "Frondicularia" rakauroana, pozo Tiburón, cuenca de Malvinas. 12, Asociación de ambiente batial, banco Maurice Erwin, plataforma de Malvinas. 13, Asociación tipo flysch, de foraminíferos aglutinados dominantes, turbiditas de la sección inferior de la Formación Policarpo, cuenca Austral, de ambientes tectónicamente activos. 14, Asociaciones de plataforma media, península Antártica. Paleogeography of the Maastrichtian transgression in the Patagonia and the Tierra del Fuego Island and distribution of the most characteristic foraminiferal assemblages in key localities. The paleocirculation after Ciesielski et al. (1977). The distribution of the land area and islands between Tierra del Fuego and the Antarctic Peninsula, is conjectural; alternatively an isthmus could replace the islands (Barker et al., 1977). 1, The northernmost assemblage constituted by simple miliolids, or with Protelphidium primitivum $n$. sp., very shallow calcareous and oolitic facies of the Saldeño and Roca Formations, Neuquina Basin. 2, Very poor assemblages, with small tests and planktonic forms only represented by opportunistic species of the genus Heterohelix, suggesting very near shore environment, Salado Basin. 3, Buliminid dominated assemblage, dysoxic dark mudstones facies, which represents the deeper environments of the northern Patagonia, Jagüel Formation, Neuquina-on shore Colorado Basin. 4, Mid-shelf benthic foraminiferal assemblage, of medium diversity, with Notoplanulina rakauroana, Pedro Luro Formation, off shore Colorado Basin. 5, Almost monospecific assemblage of Tehuelchella caminosi n. gen. n. sp., connecting area between the Colorado and the Golfo de San Jorge Basins, Aguada Cecilio Member of the Arroyo Barbudo Formation. 6, Low diversity agglutinated foraminiferal assemblage, from hyposaline deltaic environments, Lefipán Formation, Lefipán-Paso del Sapo intracratonic depocenter, Cañadón Asfalto Basin. 7 , Poor foraminiferal assemblages, Salamanca Formation, Golfo de San Jorge Basin. 8, Very low diversity assemblages of agglutinated foraminifera, hyposaline paleoenvironments, Calafate Formation. 9, Coryphostoma incrassata gigantea Assemblage, from the mid-platform glauconitic Monte Chico Formation, Austral Basin. 10, Low diversity assemblages of agglutinated, and calcareous foraminifera, dominant hyposaline environments, stable margin, Austral Basin. 11, Residual assemblage with "Frondicularia" rakauroana, Tiburón well, Malvinas Basin. 12, Bathyal assemblage, Malvinas platform, Ewing bank. 13, Flysch-type foraminiferal assemblage, lower section of the Policarpo Formation, tectonically active, turbiditic environment, Austral Basin. 14, Mid-shelf assemblages, Antarctic Peninsula. 
ficos y paleoecológicos, y aplicación en la confección del mapa paleogeográfico.

\section{LAS ASOCIACIONES DE FORAMINÍFEROS DEL MAASTRICHTIENSE PATAGÓNICO Y FUEGUINO}

Los foraminíferos bentónicos responden fuertemente a las condiciones paleoambientales, que en asociaciones de muy baja diversidad se expresan mediante el marcado dominio de algunos morfotipos. A continuación se describen las asociaciones de ambientes extremos, comenzando por las más australes.

\section{Cuenca Austral o Magallánica}

En la Cordillera Fueguina, en la parte inferior de la Formación Policarpo, asignada al Maastrichtiense por el contenido de Diplomoceras Hyatt, 1900 y Maorites Marshall, 1926, se diferencian dos asociaciones dominadas por foraminíferos aglutinados cosmopolitas de ambientes turbidíticos. Una de muy baja diversidad que se distribuye en el área de la bahía Thetis y que se caracteriza por la aparición en la cuenca de Spiroplectammina spectabilis (Grzybowski, 1898) de acuerdo con Olivero et al. (2003). La segunda, en la región del río Bueno donde alcanza un espesor mínimo de $90 \mathrm{~m}$, y se reconoce la Zona de Gaudryina healyi, con dominio de las formas aglutinadas: Thalmannammina cf. turbinata (Brady, 1881), S. spectabilis, Recurvoides sp., Gerochammina conversa (Grzybowski, 1898), y caracterizada por Silicosigmoilina angusta Turenko, 1983 y Rzehakina fissistomata (Grzybowski, 1901); entre las pocas especies calcáreas y de distribución restringida se encuentran: Charltonina acutimarginata (Finlay, 1940), Anomalinoides cf. piripaua (Finlay, 1939), y Praebulimina kickapooensis (Cole, 1938), ver Olivero et al. (2002) y Caramés \& Malumián (2006).

Hacia la plataforma, las sedimentitas maastrichtienses pierden espesor y contienen una monótona microfauna de foraminíferos aglutinados, como en el pozo ACN x-1, que atravesó un nivel glauconítico de $30 \mathrm{~m}$ de espesor con abundantes $S$. spectabilis y Rzehakina minima Cushman \& Renz, 1946 (Jannou, 2007). En la región fueguina extracordillerana es notable la extendida ausencia de foraminíferos indicadores del Maastrichtiense, incluso del Campaniense Superior y Daniense (Malumián \& Náñez, 1996).

En la Provincia de Santa Cruz, se ha ilustrado para la Formación Monte Chico, la asociación maastrichtiense de plataforma media, denominada Asociación de Coryphostoma incrassata gigantea (Wicher, 1949), de relativamente pobre preservación (Malumián \& Náñez, 2002 a,b). Sus foraminíferos más conspicuos fueron mencionados junto con Nephrolithus frequens Gorka, 1957 por Ronchi \& An- gelozzi (1994). La asociación contiene especies descritas originalmente para Nueva Zelanda, como Bolivinoides dorreeni Finlay, 1940, Bulimina rakauroana Finlay, 1940 y Dorothia biformis Finlay, 1939. En posición muy marginal sobre la plataforma estable, en el pozo SC-1, se encuentra una asociación de foraminíferos aglutinados de muy baja diversidad, excelentemente conservados, dominados por Cribrostomoides? ex gr. H. rugosus Cushman \& Waters, 1927 (ver Malumián \& Náñez, 1990) que sugiere condiciones someras hiposalinas. En el extremo septentrional de la cuenca, en la Formación Calafate, en secuencias areniscosas mayormente estériles, solo se encontraron niveles con foraminíferos aglutinados, particularmente en una asociación monoespecífica de Haplophragmoides sp. (Caramés, 1993) comparable a la anterior.

La Formación Man Aike, de la Provincia de Santa Cruz, previamente considerada maastrichtiense en análisis paleogeográficos (Malumián et al., 1983; Riccardi, 1987, 1988), es eocena media tardía (Malumián, 1990), al igual que la Formación Leticia, de Tierra del Fuego, también anteriormente asignada al Maastrichtiense. La Formación Policarpo fue considerada anteriormente Campaniense (Riccardi, 1987, 1988).

\section{Cuenca de Malvinas}

Durante el Cretácico Superior y Paleógeno, la cuenca de Malvinas evolucionó por hundimiento térmico como una cuenca de intraplaca. Particularmente durante el Maastrichtiense fue una cuenca hambrienta y sus escasos sedimentos fueron en gran parte eliminados o devastados por un proceso erosivo del Paleoceno medio (Galeazzi, 1996, 1998; Yrigoyen, 1989), reflejado en la escasez de foraminíferos maastrichtienses y danienses (Malumián \& Náñez, 1996).

Los autores verificaron la ausencia de sedimentitas maastrichtienses en varios pozos de exploración de la cuenca de Malvinas, como el Krill x-1. También se reconoce una discordancia que comprende tanto al Maastrichtiense como al Daniense, como en el pozo Ciclón x-1. Sin embargo, se ha hallado en el pozo Tiburón x-1 una secuencia de $50 \mathrm{~m}$ de espesor del Campaniense tardío-Maastrichtiense, representada mediante muestras de inyección cada $10 \mathrm{~m}$, entre 820 a 880 m bajo boca de pozo, que contiene dos asociaciones de foraminíferos separadas por un nivel glauconítico estéril entre los metros 860 y 870 . La asociación superior, entre 820 a $860 \mathrm{~m}$, en regular a buen estado de conservación, comprende entre los relativamente escasos foraminíferos bentónicos a: "Frondicularia" rakauroana (Finlay, 1939), Allomorphina sp., "Anomalinoides" rubiginosus (Cushman, 1926), Coryphostoma incrassata (Reuss, 1851), Gaudryina healyi Finlay, 1939, Notoplanulina rakauroana (Finlay, 1939), Stensioeina beccariiformis (White, 1928), y Valvulineria teuriensis Loeblich \& Tappan, 1964. Entre los muy escasos foraminíferos planctónicos se han identificado las especies Archaeoglobigerina australis Huber, 
1990, Rugotruncana circumnodifer (Finlay, 1940), y $R$. subcircumnodifer (Gandolfi, 1955).

La asociación inferior, en 870-880 m, contiene muy abundantes foraminíferos planctónicos, dominados por Archaeoglobigerina australis, secundado por Globigerinelloides impensus Sliter, 1977, Heterohelix globulosa (Ehrenberg, 1840), y Laeviheterohelix glabrans (Cushman, 1938). La especie bentónica dominante es $S$. beccariiformis, y se destaca Marginulinopsis sp. de Malumián, 1968.

De acuerdo con la zonación propuesta por Huber (1992b), la asociación inferior pertenece a la Zona de $G$. impensus, cuyo techo se encuentra en la parte superior del Cron 33N; además, L. glabrans caracteriza la parte superior de la Zona. La asociación inferior se sitúa dentro del lapso comprendido por el Campaniense medio superior al superior bajo. La asociación superior por el contenido de Rugotruncana circumnodifer, de aparición en el C32N, cuyo techo es muy próximo al límite Campaniense/Maastrichtiense, se considera maastrichtiense, posiblemente inferior por la aparente ausencia de Globotruncanella petaloidea Gandolfi, 1955 y Abathomphalus mayaroensis (Bolli, 1955). Las edades mencionadas fueron calibradas según la escala de Ogg et al. (2004).

\section{Cuenca del Golfo de San Jorge}

En esta cuenca se ha identificado el Maastrichtiense mediante foraminíferos en solo una localidad. En la base de la Formación Salamanca, en las proximidades de la localidad de Las Plumas, Provincia del Chubut, se encontró una asociación de baja diversidad, con ejemplares de pequeño tamaño, relativamente bien conservados (Náñez, 1998; Malumián \& Náñez, 2002b). De acuerdo con la presente revisión, la especie dominante entre los foraminíferos calcáreos es "Discorbis" correcta Carsey, 1926, acompañada por Buliminella isabelleana Camacho, 1954, Charltonina acutimarginata (Finlay, 1940) y Angulogavelinella? sp. La última especie, provisionalmente clasificada y bajo estudio, probablemente pertenezca a un nuevo género y está ampliamente distribuida en la cuenca Neuquina-Colorado costa adentro. Entre los pocos foraminíferos aglutinados se hallan especies de Cribrostomoides Cushman, 1910 y Trochammina Parker \& Jones, 1859. En una extensa área, conocida como el alto Camarones, no se ha identificado el Maastrichtiense marino (Masiuk, 1967), pese a la detallada búsqueda en la que ha participado uno de los autores del presente trabajo. En subsuelo solo se ha reconocido mediante nanoplancton calcáreo (Barcat et al., 1989).

\section{Área de conexión entre la cuenca del Golfo de San Jor- ge y la del Colorado-Neuquina}

En el depocentro intracratónico del Cretácico SuperiorPaleoceno de Paso del Sapo-Lefipán (Spalletti, 1996), en la Formación Lefipán, Musacchio (1995) halló la Zona de
Coryphostoma decurrens, zona local propuesta por Bertels (1980), con Buliminella isabelleana, Coryphostoma decurrens (Ehrenberg, 1854), Pyramidina prolixa (Cushman \& Parker, 1935), Eponides lunata Brotzen, 1948 y Gavelinella neuquense Bertels, 1980. En la misma Formación, los autores del presente trabajo han reconocido una de las pocas asociaciones de foraminíferos aglutinados en la Patagonia septentrional. Está conformada cuasi monoespecíficamente por Haplophragmoides sp. y otras formas planoespirales muy escasas, que son coherentes con los ambientes deltaicos descritos por Olivero et al. (1990), y reflejan condiciones hiposalinas en esta región de la $\mathrm{Pa}$ tagonia septentrional.

La Formación La Colina, en la localidad de Bajada Moreno, comprende una asociación de muy baja diversidad con una elevada cantidad de formas monoloculares calcáreas, que en algunos niveles alcanzan al $10 \%$ del total de los ejemplares, representada por Favolagena ardolinoi Malumián, Náñez \& Caramés, 1991 (ver Malumián et al., 1991).

El Miembro Aguada Cecilio, sección maastrichtiense de la Formación Arroyo Barbudo, Provincia de Río Negro, contiene una asociación de muy baja diversidad, cuasi monoespecífica, de ambiente muy somero. Sus formas calcáreas fueron mencionadas como "Pararotalia" sp. y Fissoelphidium? sp. (Malumián en Lizuaín, 1983; Malumián et al., 1995; Náñez en Caminos, 2001) y aquí se describen como Tehuelchella caminosi n. gen. n. sp., y Protelphidium primitivum n. sp., respectivamente. La primera especie también es dominante en el tramo inferior de la Formación Jagüel en el Bajo de Santa Rosa (Náñez en Hugo \& Leanza, 2001).

En esta área de conexión, principalmente ámbito de la cuenca de Cañadón Asfalto y en la que se incluye la cuenca de Península Valdez, existen afloramientos de calizas maastrichtienses con trigonias, muy discontinuos, como la Formación Puesto La Picada (Cortés, 1987), y afloramientos en la desembocadura de los Arroyos Salado y Verde, conocidos desde los trabajos pioneros sobre la Patagonia (Wichmann, 1927) y de los que no pudieron obtenerse microfósiles por su fuerte silicificación y/o recristalización del material calcáreo. Sorprendentemente, en el próximo pozo Península Valdés, si bien se encontraron típicos foraminíferos danienses, no se descubrieron foraminíferos maastrichtienses (Masiuk et al., 1976; Caramés et al., 2004).

Pese a que no se verificó la continuidad de sedimentitas maastrichtienses a través del macizo de Somún Curá, debido a la cobertura de basaltos post paleocenos, la fuerte similitud entre las asociaciones de la cuenca del Golfo San Jorge y Neuquina-Colorado costa adentro, sugiere una conexión entre ambas. Los numerosos afloramientos reconocidos en el relevamiento regular, indican que el macizo fue inundado por la transgresión (Page et al., 1999). 


\section{Cuenca Neuquina-Colorado costa adentro}

La cuenca Neuquina-Colorado costa adentro cobijó numerosos foraminíferos endémicos.

Se diferencian principalmente cinco asociaciones de foraminíferos calcáreos. Tres son de muy baja diversidad: (1) una, en los términos basales de la Formación Jagüel, dominada por glandulínidos, discórbidos y frecuentes polimorfínidos (Kielbowicz, 1980; Náñez \& Concheyro, 1997). Otra (2), en la misma Formación pero en facies más arenosas, en la localidad Liu Malal (Pires et al., 1999), dominada fuertemente por formas trocoespiraladas pequeñas con botón umbilical, y con relativamente frecuente Rosalina patagonica n. sp. y, raro, Protelphidium primitivum n. sp. (3) La tercera, en la Formación Roca, es la asociación conocida de posición más septentrional (Náñez en Parras et al., 1998), con ejemplares escasos y de regular a mala preservación por recristalización; conformada por rotálidos, relativamente abundante P. primitivum n. sp. y presencia de Tehuelchella n. gen., y caracterizada por un par de especies de miliólidos indeterminables, compatibles con el carácter calcáreo de la Formación. Las extremas condiciones que refleja esta asociación sugieren que el límite septentrional alcanzado por la transgresión se halla próximo.

La transgresión maastrichtiense sobrepasó el límite norte de la Patagonia alcanzando $c a .33^{\circ}$ de latitud sur. Se reconoce así hasta dentro de la actual alta Cordillera de Mendoza en cotas superiores a los 3.500 metros de altura, representada por los términos superiores de la Formación Saldeño, con calcáreos oolíticos y estromatolíticos, y una asociación (4) de miliólidos identificados en secciones delgadas (Polanski, 1964; Tunik, 2003).

La asociación calcárea (5) más diversificada de la cuenca Neuquina-Colorado costa adentro comprende varias y abundantes especies descritas por Bertels (1972, 1980) que aparentaban ser endémicas. Se trata en general de una asociación típica de ambientes disaeróbicos, dominada por bulimínidos de pequeño porte contenida en limoarcilitas oscuras, carentes o con escasa megafauna conchífera, de los términos medios de la Formación Jagüel. Los valores de diversidad del índice $\alpha$ en los últimos $11 \mathrm{~m}$ de sedimentitas maastrichtienses en el perfil de Bajada de Jagüel oscilan entre 4 y 9. La especie más distintiva por su abundancia, particular morfología, y distribución austral es Hiltermannella kochi (Bertels, 1970).

Una particular asociación con foraminíferos aglutinados, confinada a la base del perfil de Bajada de Jagüel de la Formación Jagüel, contiene abundante $S$. spectabilis, en una presentación típica de esta especie "desastre".

\section{Cuenca del Colorado costa afuera}

En el pozo Pejerrey, costa afuera de la cuenca del Colorado, reconocemos una asociación inédita de carácter Austral. Esta asociación contiene: Alabamina creta (Fin- lay, 1940), Allomorphina cretacea Reuss, 1851, Coryphostoma incrassata gigantea, Gavelinella neuquense, Notoplanulina rakauroana, Praebulimina carseyae (Plummer, 1931), Quadrimorphina camerata (Brotzen, 1936), Stensioeina beccariiformis, con cerca de un tercio de especies aglutinadas entre las que se encuentran: Dorothia bulletta (Carsey, 1926), Reticulophragmium sp., Rzehakina fissistomata y Trochammina sp.

\section{LOS TAXONES DE MAYOR RELEVANCIA PALEOBIOGEOGRÁFICA}

En la cuenca Neuquina-Colorado costa adentro, para el Maastrichtiense se han descrito varias especies (Bertels, 1972, 1980), en principio aparentemente endémicas de la cuenca; no obstante, tras una revisión (ver comentarios sistemáticos) se excluyen aquellas consideradas variantes locales o probables sinónimos de especies cosmopolitas. Se estima que solo las siguientes especies son hasta la fecha endémicas del Hemisferio Sur: Buliminella pseudoelegantissima Bertels, 1972, Charltonina acutimarginata, Favolagena ardolinoi, Hiltermannella kochi, Lagenoglandulina neuquensis (Bertels, 1980), Neobulimina argentinensis Bertels, 1972, у Praeglobobulimina jaguelensis Bertels, 1972.

Las especies endémicas que originalmente se describieron para el Daniense y luego se reconocieron también en el Maastrichtiense de la Patagonia son: Favolagena atilai (Bertels, 1964) y Buliminella isabelleana Camacho, 1954, forma tuberculata Malumián \& Caramés, 1995.

Estas especies son comentadas a continuación, resaltándose los rasgos propios que conforman el común denominador de las faunas australes.

Buliminella pseudoelegantissima es de fuerte carácter oportunista como su homeomorfo actual B. elegantissima (d'Orbigny, 1839). Se distribuye desde el Maastrichtiense, sobrevive al límite Cretácico/Paleógeno (K/Pg), con su mayor abundancia en las fracciones más finas del Daniense basal (Náñez \& Malumián, 2004) y aparentemente subsiste hasta sus términos más altos. Fuera de la Patagonia septentrional, solo ha sido ilustrada en ambientes profundos del Campaniense tardío de la cuenca de Sergipe, donde es muy rara (Koutsoukos \& de Klasz, 2000).

Buliminella isabelleana forma tuberculata es una forma típica de la cuenca Neuquina-Colorado, y parte del plexo de Buliminella westralensis Parr, 1938 (ver Malumián \& Caramés, 2002).

Charltonina acutimarginata $=$ Alabamina kaasschieter Bertels, 1980. La sinonimia de esta especie en la Argentina está sostenida por su continua distribución desde la cuenca Austral, cuenca del Golfo y Patagonia septentrional. 
Hiltermannella kochi puede considerarse como uno de los taxones provinciales australes emblemáticos. La concha es pequeña, y por sus cámaras con una fuerte indentación basal y en disposición biserial torsionada semeja en su morfología general al género Gabonita, según fue puntualizado por Bertels (1970). Es particularmente próxima y puede considerarse homeomorfa de algunas de las especies lisas descritas posteriormente para el Maastrichtiense de Egipto (El-Shinnawi, 1972), como G. articulata El-Shinnawi, 1972. Pero Hiltermannella, por su abertura en forma de coma y con placa dental se diferencia netamente de $\mathrm{Ga}$ bonita, un género muy variable en su morfología general, caracterizado por una abertura en forma de herradura, de contorno entero, y carente de placa dental. La ausencia de placa dental llevó a Odébòde (1987) a proponer la familia Gabonitidae. Si bien Gabonita tiene una amplia distribución con especies reconocidas en el CenomanienseTuroniense de Sergipe, Brasil, Gabón, Marruecos, Egipto e Israel (Koutsoukos, 1992), no se ha encontrado en la Argentina, ni en las asociaciones australes.

En la comparación general de ambos géneros se destacan aspectos paleoecológicos similares que explicarían la similitud morfológica externa. Gabonita es un género euritópico y constituye asociaciones oportunistas prácticamente monoespecíficas en el Gabón. Su aparente dificultad en habitar ambientes de mar abierto o de aguas profundas explicaría la limitada y discontinua distribución biogeográfica: no ha sido encontrado en el Coniaciense-Maastrichtiense de Sergipe, y solo muy raros ejemplares se han reconocido en el Cretácico de las cuencas marginales ecuatoriales del Brasil (Koutsoukos \& de Klasz, 2000).

La distribución de $H$. kochi es un caso ilustrativo de una aparente discontinuidad en su distribución geográfica, dado que es abundante en el Maastrichtiense de la Patagonia septentrional y más aún en la península Antártica (Huber, 1988), pero no se ha reconocido en las cuencas Austral y de Malvinas. Esta aparente restricción puede deberse en gran parte al desconocimiento de los foraminíferos en ambas cuencas sureñas a causa del escaso remanente de depósitos marinos maastrichtienses, o alternativamente, a la ausencia de paleoambientes propicios. Por otra parte, Huber (1988) notó que Pseudouvigerina cretacea de Webb (1971), de Nueva Zelanda, puede pertenecer a esta especie, que cobraría así una clara distribución austral; pero la suposición no pudo verificarse mediante la comparación directa del material. Se reconocieron pocos caparazones de $H$. kochi por encima del límite $\mathrm{K} / \mathrm{Pg}$ en Patagonia septentrional que sugieren que es una especie de extinción aplazada, aunque no puede descartarse el retrabajo de las conchas.

Favolagena atilai es un foraminífero unilocular calcáreo sumamente particular por su variación morfológica: desde una cámara elongada en el Maastrichtiense hasta con la cámara más ancha que alta, en el Daniense. Hasta aho- ra ha sido reconocido en la Patagonia septentrional y en la cuenca Austral (Caramés \& Malumián, en Malumián \& Panza, 2000).

Lagenoglandulina neuquensis es una especie que se reconoce también en el Daniense y pertenece a un género bien representado hasta el Eoceno medio, tanto en Nueva Zelanda como en Tierra del Fuego.

Protelphidium primitivum n. sp. y Rosalina patagonica n. sp., son los representantes más antiguos de sus respectivos géneros; como especies no se han encontrado en el Daniense de la Patagonia.

Tehuelchella caminosi $\mathrm{n}$. gen. $\mathrm{n}$. sp. es casi la única especie en niveles de cuatro metros de espesor del Miembro Aguada Cecilio, en el área de laguna del Indio Muerto. Sumamente abundante, se recuperaron más de 6.000 ejemplares en 100 gr de muestra. La bajísima diversidad indica un ambiente sumamente estresado y marginal reflejado también por el crecimiento algo irregular, claramente manifiesto en el lado espiral de las conchas. En el bajo de Santa Rosa, también conforma asociaciones cuasi monoespecíficas. En el área de Aguada Cecilio, está asociada a Protelphidium primitivum n. sp. y abundantes miliólidos. También se ha reconocido en las pobres asociaciones del extremo septentrional de la cuenca Neuquina. Si bien Tehuelchella no se reconoce en el Daniense, pertenece a un tipo morfológico abundante y distribuido en la Patagonia desde el Eoceno superior, pues en líneas generales recuerda al género Buccella Andersen,

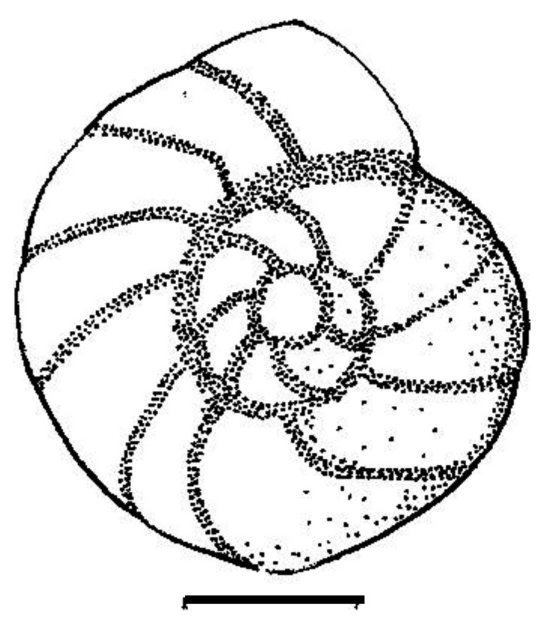

Figura 3. Dibujo esquemático del lado espiral de un paratipo de Tehuelchella caminosi n. gen. n. sp., con aumento anómalo del tamaño de las cámaras. Ejemplar de Laguna del Indio Muerto. La barra equivale a 100 $\mu \mathrm{m}$. SEGEMAR 2824.

Sketch of the spiral side of a paratype of Tehuelchella caminosi n.gen.n. sp., showing chamber anomalous size increase. Specimen from Laguna del Indio Muerto. Scale bar represents 100 Hm. SEGEMAR 2824. 


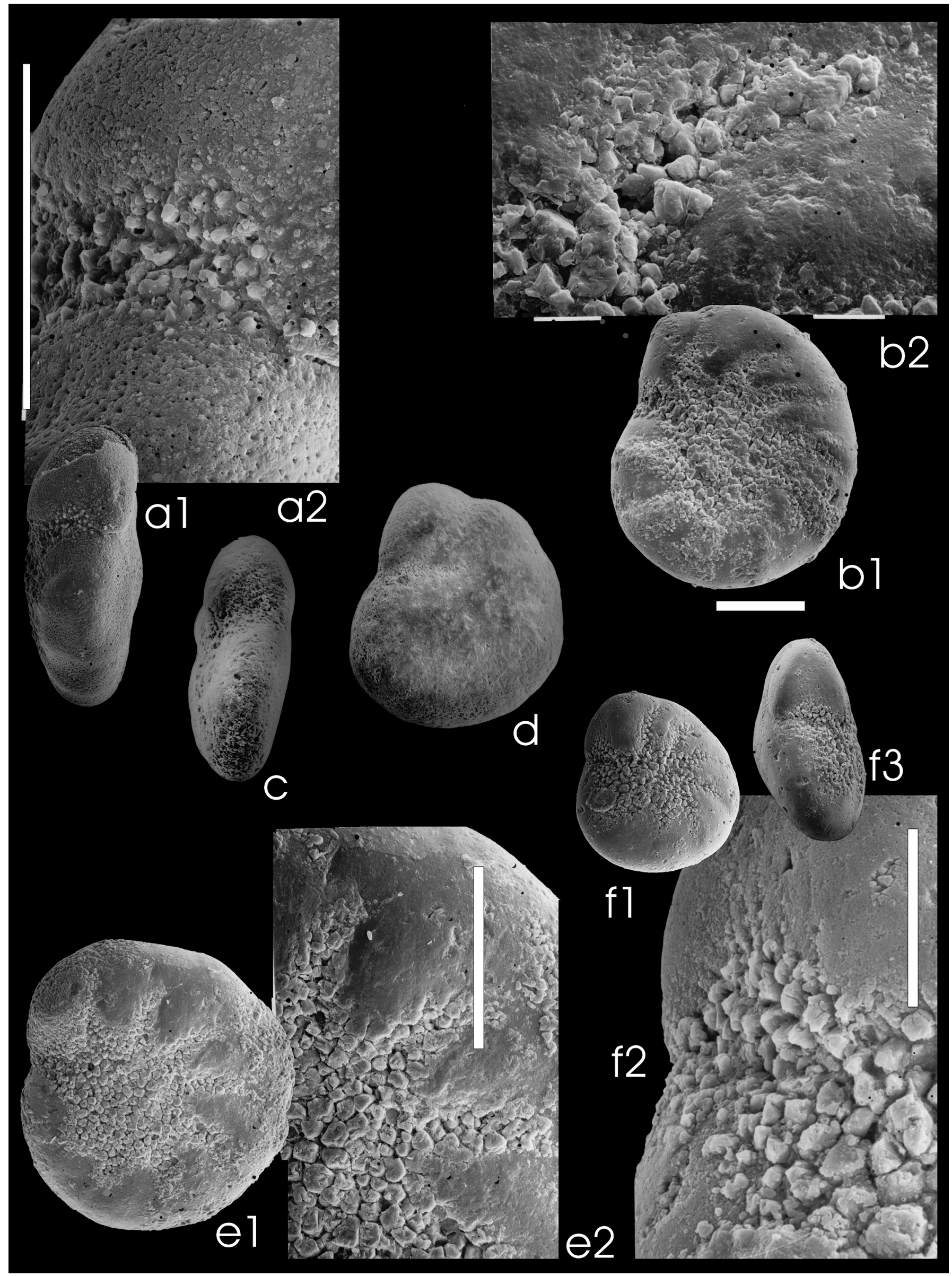


1952 y formas similares, que en oportunidades se han diferenciado bajo la denominación de "Buccella" (Malumián, 1982; Caramés et al., 2004). Este tipo morfológico está caracterizado por una región umbilical tuberculada, que se extiende por las suturas hasta la periferia, y un lado espiral muy liso. La abundancia de Buccella, en la asociación de la actual plataforma argentina, llevó a Boltovskoy (1976) a denominarla como el Reino de Buccella. Por otra parte, en general las formas con superficie tuberculada son las más características de la región austral (Malumián, 1994), y se ha sugerido que esta superficie pueda ser un medio de defensa para evitar la predación mediante perforaciones (Malumián et al., 2007).

"Frondicularia" rakauroana y Notoplanulina rakauroana son especies australes que pertenecen a géneros muy conspicuos, no reconocidos en la Patagonia septentrional. "Frondicularia" rakauroana es sumamente característica por su morfología, distribución cronológica y paleobiogeografía. Su morfología destaca por la fuerte escultura y gran tamaño, ambas características inusuales en las conchas de altas latitudes. Si bien se la ha reasignado al género Frondicularia Defrance, 1826, por su gran tamaño y fuerte escultura hallaría mejor encasillamiento dentro de Neoflabellina Bartenstein, 1948, ya que todos los ejemplares conocidos son megalosféricos y podrían, por el tipo de generación, carecer del inicio planoespiral que identifica al último género. Su distribución cronológica se restringe al Maastrichtiense superior en Nueva Zelanda, y se ha encontrado en el Maastrichtiense de la cuenca Carnarvon de Australia y en la elevación de Lord Howe (Huber \& Webb, 1986; Huber, 1992a). Su mención en el Campaniense superior de la península Antártica, incluso en una muestra junto con abundante H. kochi (Huber, 1984, 1988), debe ajustarse al Maastrichtiense inferior, pues se distribuye por encima de niveles con Gunnarites Kilian \& Reboul, 1969 (Crame et al., 1999; Olivero \& Medina, 2000). El hallazgo en la cuenca de Malvinas, extiende su límite septentrional hasta cerca de $54^{\circ} \mathrm{LS}$.

Notoplanulina rakauroana $=$ Planulina rakauroana Finlay, 1939, es una especie sumamente característica y ampliamente distribuida en medianas altas latitudes del Hemisferio Sur desde el Santoniense hasta el Campaniense inferior. Aparentemente su distribución geográfica se restringe en el Maastrichtiense superior, desplazándose hacia latitudes algo menores. Solo se verifica su presencia en el Maastrichtiense superior, o sea dentro de la Zona de Abathomphalus mayaroensis, en Nueva Zelanda y en la elevación de Lord Howe (Webb, 1971, 1973). Es relativamente abundante en el Santoniense-Campaniense inferior de Kerguelen y también se reconoce en el Maastrichtiense inferior (Quilty, 1992). En el sitio 1138, se distribuye hasta el Campaniense superior (Quilty, 2002). Pero Kaiho (1998) le asigna un biocrón Coniaciense-Campaniense (88-72Ma) basado en la distribución reconocida en el sitio DSDP 258, y considera que se origina de Notoplanulina primitiva Kahio, 1998, del Turoniense.

Fuera de Australasia, está asociada a ammonites del Santoniense superior-Campaniense inferior (Malumián, 1990) de la cuenca Austral; para el Campaniense del plateau de Malvinas ha sido ilustrada bajo la denominación de Gavelinella compressa Sliter, 1968, por Basov \& Krasheninnikov (1983: lám. 10, figs. 2-4; Malumián \& Náñez, 1996). En la cuenca de Malvinas tiene su última aparición en el Maastrichtiense inferior, reconociéndose en el pozo Tiburón con ejemplares que alcanzan a un milímetro de diámetro. No se encontró en la Formación López de Bertodano (Huber, 1988), Maastrichtiense superior temprano (Olivero \& Medina, 2000) de la isla Seymour de la Antártida.

En cuenca del Colorado costa afuera, grandes ejemplares están asociados a Coryphostoma incrassata gigantea, sugiriendo una edad maastrichtiense media. En los sitios 363 y 364 del DSDP Leg 40, ha sido ilustrada como $G a$ velinella compressa, restringida al Campaniense inferior, y al Santoniense, y son los registros de menor latitud, entre 20 y $10^{\circ} \mathrm{LS}$, que junto con otras formas australes conforman una asociación interpretada como mixta (Beckmann, 1978); según la deriva de África hacia el norte, la paleolatitud debe considerarse con una decena de grados más.

Si bien el género Notoplanulina Malumián \& Masiuk, 1976 es morfológicamente una singularidad (Revets, 1996), encuentra homeomorfos modernos en Planulina ariminensis d'Orbigny, 1826, y formas discoidales comprimidas como "Cibicides" wuellerstorfi (Schwager, 1866), taxones epifaunales que prefieren una posición elevada respecto a la interfase sedimento-agua y por lo tan-

Figura 4. Tehuelchella caminosi n. gen. n. sp. Ejemplares de Laguna del Indio Muerto, excepto c y d, provenientes del Bajo de Santa Rosa. a1, vista periférica panorámica; a2, detalle de la base de la última cámara cubierta por tubérculos, SEGEMAR 2825. b, Holotipo; b1, vista umbilical; b2, detalle de la sutura, SEGEMAR 2826. c, Vista periférica, SEGEMAR 2827. d, Vista espiral, SEGEMAR 2828. e1, vista umbilical; e2, detalle del área umbilical y suturas, SEGEMAR 2829. f1, Vista umbilical; f2, detalle de la base de la última cámara; f3, vista periférica, SEGEMAR 2830. La barra representa $100 \mu \mathrm{m}$. Tehuelchella caminosi $n$. gen. n. sp. Specimens from Laguna del Indio Muerto, except c and d, from Bajo de Santa Rosa. $\boldsymbol{a 1}$, peripheral view; $\boldsymbol{a} 2$, detail of the last chamber base covered by tubercles, SEGEMAR 2825. b, Holotype; b1, umbilical view; b2, detail of the suture, SEGEMAR 2826. c, Peripheral view, SEGEMAR 2827. d, Spiral view, SEGEMAR 2828. $\boldsymbol{e 1}$, umbilical view; $\boldsymbol{e 2}$, detail of the umbilical area and sutures, SEGEMAR 2829.f1, umbilical view; f2, detail of the last chamber base; f3, peripheral view, SEGEMAR 2830. Scale bar represents 100 $\mathrm{m}$. 


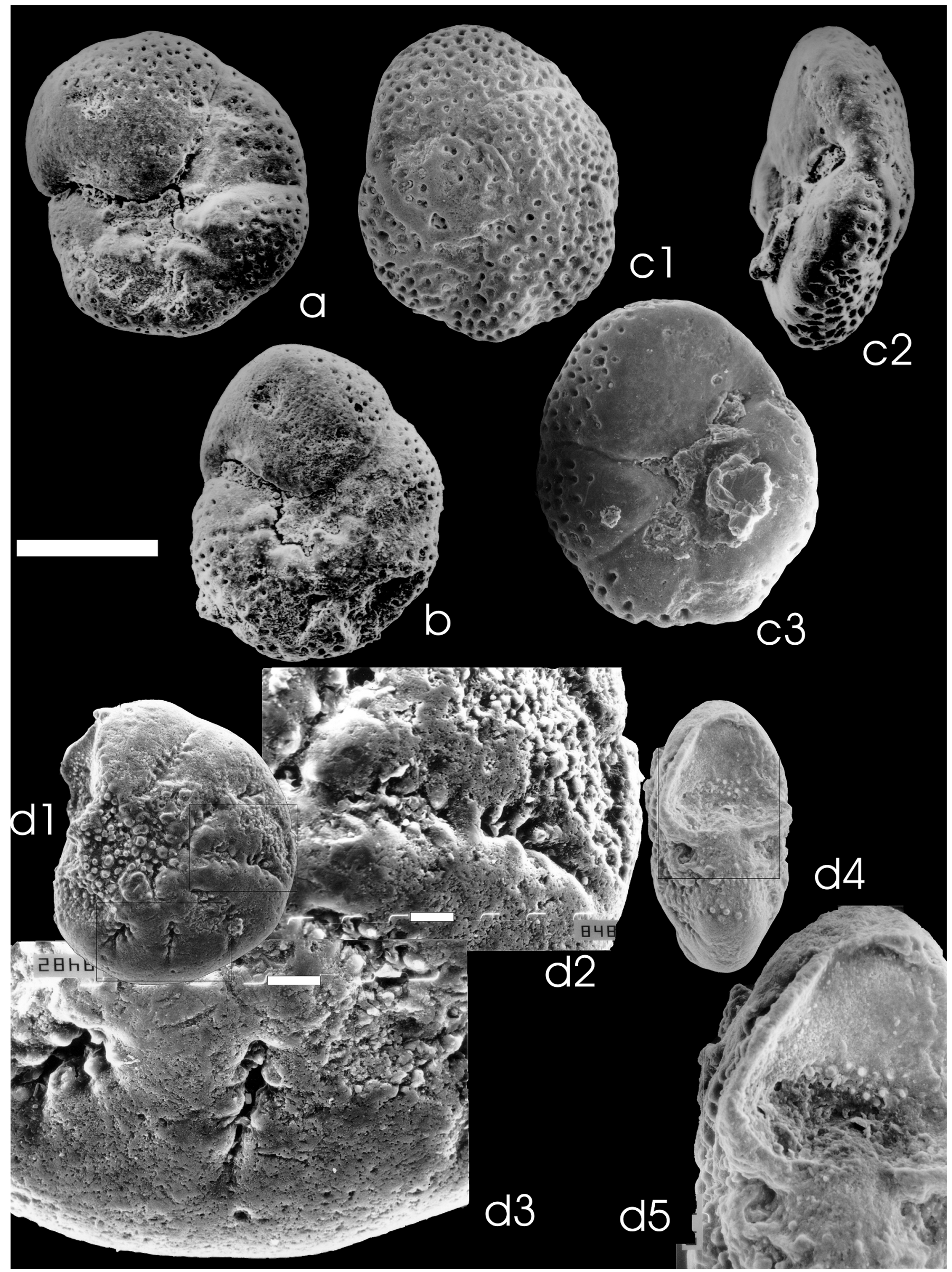


to son suspensívoros pasivos (Lutze \& Thiel, 1989). Las conchas de $N$. rakauroana tienden a aumentar de tamaño a menores edades; las formas premaastrichtienses son relativamente pequeñas y en general menores a $850 \mu \mathrm{m}$ de diámetro, mientras que las maastrichtienses alcanzan el milímetro.

\section{DISCUSIÓN Y CONCLUSIONES}

\section{Paleobiogeografía}

El progreso sobre el conocimiento de los foraminíferos patagónicos del Maastrichtiense en la última década modifica la visión previa - tanto en el ámbito local, como dentro de la Provincia Austral- en la discriminación biogeográfica de las asociaciones.

En el ámbito local, debe considerarse la gran disimilitud de las características ambientales entre las cuencas de la Patagonia septentrional y la Austral, que explicaría las diferencias en las asociaciones de foraminíferos bentónicos.

La cuenca Neuquina, de antepaís, en el Maastrichtiense se encuentra en un estadio avanzado de sineclisa, caracterizada por una reducida tasa de hundimiento (Uliana \& Dellapé, 1981), y se emplaza en un área de condiciones semi-áridas comparable con el golfo Pérsico (Barrio, 1990a,b), con una probable circulación antiestuarina, condiciones extensibles a la cuenca del Colorado costa adentro. Consecuentemente, la sedimentación comprende la acumulación de calizas biogénicas a evaporíticas. Conjuntamente con la cuenca del Golfo de San Jorge, conforman un ambiente singular por su gran extensión y escasa profundidad, con espesores del Maastrichtiense marino reducidos a una veintena de metros, y dominio de asociaciones de foraminíferos calcáreos de baja diversidad. Los nuevos taxones propuestos confirman la endemicidad y perfilan su caracterización. Como entidad biogeográfica tiene su límite hacia los ambientes relativamente más profundos situados costa afuera. Solo localmente, se acumulan depósitos que alcanzan algunas centenas de metros de espesor, como en el extremo septentrional de la cuenca Neuquina (Parras et al., 1998), o en la Formación Lefipán (Olivero \& Medi- na, 1994); en esta última, asociaciones de baja diversidad dominadas por foraminíferos aglutinados planoespirales reflejan los ambientes deltaicos e hiposalinos.

La cuenca Austral, en contraste, tiene posición de retroarco, con sedimentación netamente clástica. En la Cordillera Fueguina, el Maastrichtiense contiene relativamente profundas y espesas secuencias de turbiditas (mínimo 90 m en río Bueno, y 210 m en bahía Thetis; Olivero et al., 2003); mientras que las áreas de plataforma, incluidas las de la cuenca de Malvinas, se encuentran hambrientas, dando lugar a secuencias condensadas que en muchos casos carecen de foraminíferos o tienen pobres asociaciones residuales debido a la mala y/o diferencial preservación. En el Maastrichtiense comienzan las acumulaciones de carbón en el área carbonífera de Río Turbio, que junto con el contenido en materia orgánica de las formaciones del Cretácico Superior de la Cordillera Fueguina, sugieren el inicio de un clima húmedo y de una probable circulación estuarina que se establecerá definidamente en el Paleógeno, con el consiguiente deterioro de la salinidad y proporcional aumento y abundancia de foraminíferos aglutinados en diferentes tipos de asociaciones. Estas asociaciones comprenden desde la denominada tipo flysch hasta conjuntos de muy baja diversidad propios de ambientes con influencia fluvial.

El reconocimiento de elementos típicamente australes costa afuera de la cuenca del Colorado, en ambientes más profundos que los imperantes tierra adentro, replantea la interpretación biogeográfica y cuestiona en cierto grado las consideraciones sobre el efecto de barreras topográficas. De hecho, existe una marcada diferencia batimétrica entre las asociaciones de las cuencas Neuquina-Colorado costa adentro y San Jorge, y la asociación de cuenca del Colorado costa afuera. Las dos primeras, de baja diversidad y de muy escasos foraminíferos aglutinados, en ambientes someros, y la última, con característicos foraminíferos de aguas profundas y relativamente abundantes aglutinados y planctónicos. Similares asociaciones a la de cuenca del Colorado costa afuera se han reconocido en paleolatitudes bajas costa afuera del África en el Santoniense y Campaniense (Beckmann, 1978), que sugieren una mayor distribución geográfica de los taxones australes para épocas pre-maastrichtien-

Figura 5. a-c. Rosalina patagonica n. sp. Todos los ejemplares de Liu Malal. a, b, Vista umbilical de diferentes paratipos, SEGEMAR 2831-2832. c, Holotipo; c1, vista espiral; c2, vista periférica; c3, vista umbilical, SEGEMAR 2833. d, Protelphidium primitivum $\mathrm{n}$. sp., holotipo, localidad Liu Malal, d1, vista lateral; d2, detalle de las suturas hendidas y cubiertas en la región periumbilical por material calcáreo secundario convirtiéndose en un conducto; d3, detalle de las suturas hendidas con incipientes puentes; d4, vista periférica; d5, detalle de la vista periférica con foramen en la base del septo, SEGEMAR 2834. La barra representa $100 \mu \mathrm{m}$ en las vistas panorámicas.

a-c. Rosalina patagonica $\mathrm{n}$. sp.; all specimens from Liu Malal. $\boldsymbol{a}, \boldsymbol{b}$, umbilical view of different paratypes, SEGEMAR 2831-2832. c, holotype; c1, spiral view; c2, peripheral view; c3, umbilical view, SEGEMAR 2833. d, Protelphidium primitivum n. sp., holotype, from Liu Malal, $\mathbf{d 1}$, side view; $\mathbf{d 2}$, detail of the incised sutures covered in the periumbilical region by secondary calcareous material becoming a canaliculate duct; $\mathbf{d} 3$, detail of the incised sutures with incipient ponticuli; $\boldsymbol{d 4}$, edge view; $\mathbf{d 5}$, detail showing the foramen at the base of the septum, SEGEMAR 2834. Scale bar represents $100 \mu m$ in the panoramic views. 


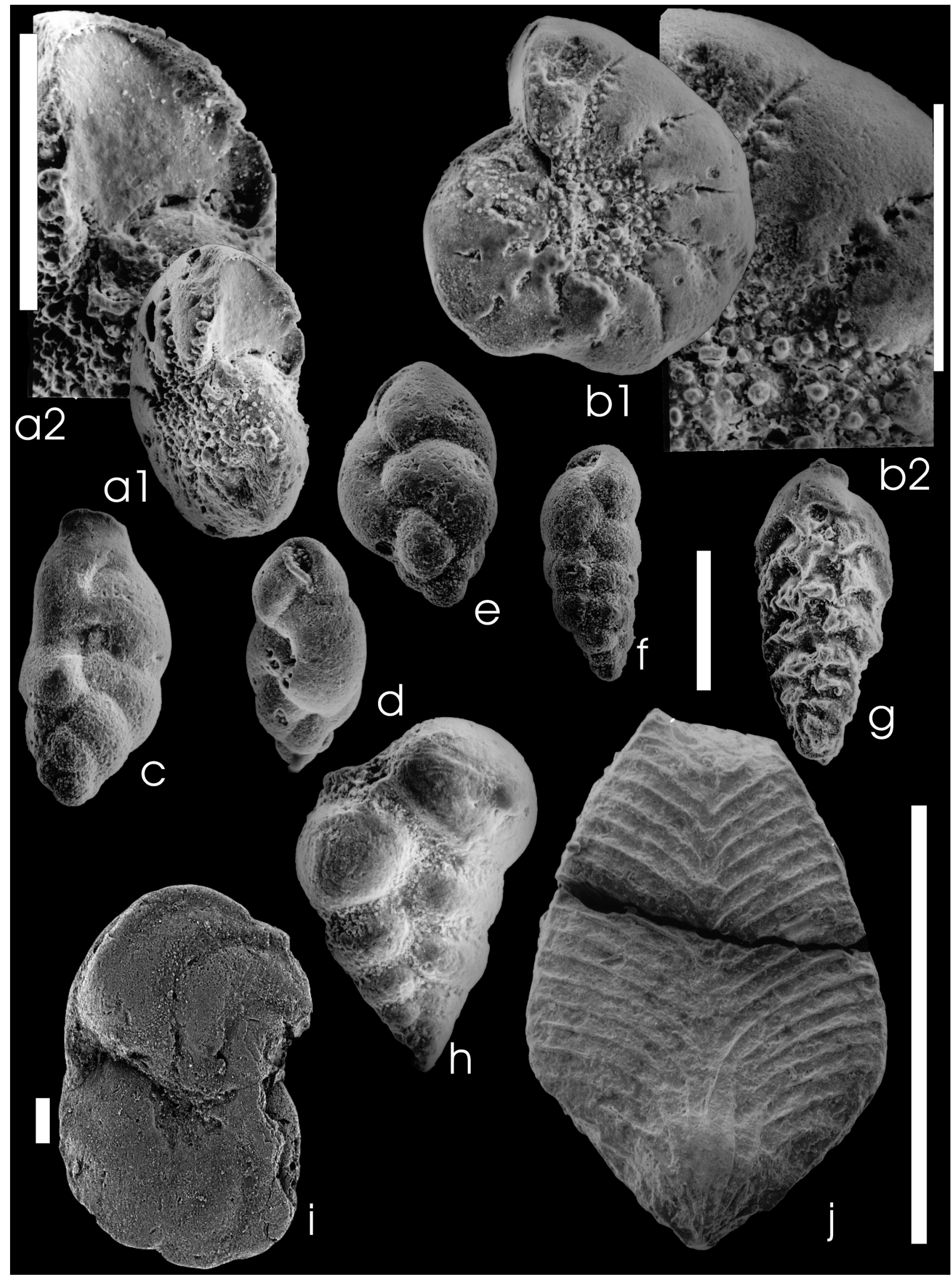


ses, y confirma la conocida capacidad de los foraminíferos bentónicos en sortear barreras, y por ende, salvo casos muy evidentes, de señalar conexiones entre cuencas.

Entre los foraminíferos bentónicos, se destacan géneros cuyos aparentes registros de máxima antigüedad se dan en el Hemisferio Sur, fuera de las regiones tropicales, y que sugieren un origen polar austral, como Lagenoglandulina Silvestri, 1923, Protelphidium Haynes, 1956, Rosalina d'Orbigny, 1826 y el plexo morfológico encabezado por Tehuelchella $\mathrm{n}$. gen. También la familia Elphidiidae tiene antiguos antecedentes en las regiones australes tanto con formas trocoespiraladas como planoespiraladas, tan antiguas como el Eoceno basal en Tierra del Fuego, en donde se reconocen los registros más antiguos de Elphidium Montfort, 1808 y de Cribrorotalia Hornibrook, 1961, en la Formación Punta Noguera. Los diferentes datos moleculares del grupo Ammonia-Elphidium respecto al resto de los rotálidos (Fahrni \& Pawlowski, 1995) sugerirían un distinto origen.

\section{Paleogeografía}

Respecto a los bien conocidos altos topográficos entre las cuencas de la Patagonia septentrional y la cuenca Austral, que incluso penetran profundamente en la plataforma continental (Fig. 1), su extensión y tamaño depende de la interpretación: si el Maastrichtiense marino fue depositado y luego erosionado por un pulso daniense, o simplemente que no se haya depositado donde hoy no se encuentra, como en las cuencas costa afuera.

Respecto a la conexión entre el océano Atlántico y el Pacífico, no pueden descartarse comunicaciones someras entre los océanos tanto a la latitud de Quiriquina, como a través de la paleo Cordillera Fueguina-península Antárti- ca. Para verificar o desechar la primera, urge un estudio de microfósiles calcáreos de la Formación Quiriquina, particularmente una comparación con los bien conocidos ostrácodos de Patagonia septentrional. Para la segunda, la similitud faunística con Australasia apoyaría una conexión pacífica, que alternativamente puede ser justificada por brazos marinos transantárticos. Es muy probable que la inexistencia de alguna de las dos, apoye la existencia de la otra: la distribución de una misma especie de Belonostomus, a ambos lados de la región andina, difícilmente puede explicarse para un organismo no pelágico, si no es con una conexión al menos somera, la cual puede haber ocurrido en la compleja historia del arco de Scotia en momentos de nivel de mar alto.

Las reconstrucciones referidas al Maastrichtiense del arco de Scotia, deberán contemplar las fuertes disimilitudes entre las asociaciones de la Formación Policarpo, vinculada a un ambiente tectónicamente muy activo; de la asociación maastrichtiense conocida para la península Antártica en la cuenca James Ross, que representa condiciones de plataforma media y externa (Huber, 1988); y del banco Maurice Ewing, de ambiente batial.

La transgresión maastrichtiense cubrió una superficie mayor que la del mar daniense (Malumián, 1999) en las cuencas Neuquina y Austral, y algo menor en la cuenca del Golfo de San Jorge. La isla resultante del área positiva desde Camarones hasta la Península Valdés, y quizás incluyendo la cuenca de Rawson, tuvo una mayor extensión que en el Daniense, posiblemente debido a algún basculamiento del macizo de Somún Curá. Este macizo fue totalmente inundado (Page et al., 1999), por lo cual una transgresión atlántica alcanzó así, una cuenca andina; pero se carece de

Figura 6. Foraminíferos representativos del Maastrichtiense de la Patagonia y la plataforma continental adyacente. La barras representan $100 \mu \mathrm{m}$, excepto en $\mathbf{j}$ donde equivale a $1 \mathrm{~mm}$. La barra en $\mathbf{f}$ es la escala para $\mathbf{c}-\mathbf{h}$. $\mathbf{a}-\mathbf{b}$, Protelphidium primitivum $\mathbf{n}$ sp., ejemplares de Liu Malal; a, vista periférica y detalle de un paratipo sin las dos últimas cámaras mostrando el foramen en la base del septo, SEGEMAR 2835; b, vista lateral de un paratipo y detalle de las suturas con puentes incipientes, SEGEMAR 2836. c-e, Hiltermannella kochi (Bertels, 1970), ejemplares de Bajada del Jagüel, Formación Jagüel, ilustrando la gran variación morfológica de la especie, SEGEMAR 2837-2839; c, ejemplar con un cuello desarrollado; d, ejemplar que muestra la abertura en forma de ojal y no enteramente rodeada por un labio. f, Neobulimina argentinensis Bertels, 1972, Formación Jagüel, Bajada del Jagüel, SEGEMAR 2840. g, Tappanina? sp., Formación Jagüel, Bajada del Jagüel, SEGEMAR 2841. h, Laeviheterohelix glabrans (Cushman, 1938), pozo Tiburón x-1, 870-80 metros bajo boca de pozo, SEGEMAR 2842. i, Notoplanulina rakauroana (Finlay, 1939), pozo Pejerrey, 1823 metros bajo boca de pozo; ejemplar de gran tamaño, SEGEMAR 2843. j, “Frondicularia” rakauroana (Finlay, 1939), pozo Tiburón x-1, 850-60 metros bajo boca de pozo, SEGEMAR 2844.

Representative foraminifera from the Maastrichtian of the Patagonia and the adjacent continental platform. Scale bar represents $100 \mu \mathrm{m}$ except in $\boldsymbol{j}$ where equals $1 \mathrm{~mm}$; bar in $\boldsymbol{f}$ is the scale for $\boldsymbol{c}-\boldsymbol{h}$. $\boldsymbol{a}-\boldsymbol{b}$, Protelphidium primitivum $n$. $s p$., specimens from Liu Malal; a, Peripheral view and detail of a paratype without the last two chambers showing the foramen at the septum base, SEGEMAR 2835; b, Lateral view of a paratype and detail of the incised sutures with incipient ponticuli, SEGEMAR 2836. c-e , Hiltermannella kochi (Bertels, 1970), specimens from Bajada del Jagüel, Jagüel Formation, showing a great morphological variability, SEGEMAR 2837-2839; , specimen with a developed neck; $\boldsymbol{d}$, specimen showing a loop shaped aperture and not entirely surrounded by a lip, Jagüel Formation. f, Neobulimina argentinensis Bertels, 1972, Jagüel Formation, Bajada del Jagüel, SEGEMAR 2840.g, Tappanina? sp., Jagüel Formation, Bajada del Jagüel, SEGEMAR 2841. h, Laeviheterohelix glabrans (Cushman, 1938), Tiburón x-1 well, 870-80 meters below surface, SEGEMAR 2842. $\boldsymbol{i}$, Notoplanulina rakauroana (Finlay, 1939), Pejerrey well, 1823 meters below surface; specimen of large size, SEGEMAR 2843. j, "Frondicularia" rakauroana (Finlay, 1939), Tiburón x-1 well, 850-60 meters below surface, SEGEMAR 2844. 
argumentos biogeográficos para apoyar la comunicación entre el sur argentino y las áreas caribeñas o peruanas.

Por el momento no puede darse una razón valedera que indique si la inundación de la cuenca del Golfo de San Jorge se produjo por el este, por la vertiente atlántica, o por el norte, a través de la cuenca de Cañadón Asfalto, dada la barrera del Alto Patagónico Oriental (Fig. 2).

El endemismo más localizado de la cuenca NeuquinaColorado costa adentro, respecto al marcado endemismo austral de las cuencas Austral y de Malvinas, podría explicarse por una forma cerrada hacia el Pacífico de la primera; mientras que para el Maastrichtiense la cuenca Austral no habría sufrido la actual fuerte flexura hacia el Océano Atlántico, sino que se abriría ampliamente hacia el océano Austral. La similar paleolatitud con Nueva Zelanda y Australia meridional (Lawver et al., 1992) explican las afinidades microfaunísticas.

\section{CONCLUSIONES GENERALES}

Las asociaciones de foraminíferos bentónicos maastrichtienses de la Patagonia y de la plataforma continental argentina bien pueden reconocerse positivamente mediante aspectos que le son propios, más que por caracteres negativos como la ausencia de taxones filotérmicos o baja diversidad. Son marcadamente diferentes de aquellas del norte de América del Sur, y se consideran pertenecientes al reino Austral, con Patagonia septentrional como centro endémico en un mar marginal. No se han encontrado manifestaciones de un brazo marino que conecte el sur de América del Sur, con las regiones peruanas y/o caribeñas.

Entre los taxones que caracterizan las asociaciones maastrichtienses australes, reconocidas en Patagonia y la plataforma continental argentina, se encuentran Hiltermannella kochi, Favolagena ardolinoi, Lagenoglandulina neuquensis, Neobulimina argentinensis, Charltonina acutimarginata, "Frondicularia" rakauroana y Notoplanulina rakauroana, a las que se agregan nuevos taxones: Tehuelchella caminosi n. gen. n. sp., Protelphidium primitivum n. sp. y Rosalina patagonica n. sp. La morfología general de Tehuelchella caminosi n. gen. n. sp., taxón que caracteriza las asociaciones maastrichtienses más someras de Patagonia septentrional, persiste hasta las asociaciones modernas de las costas patagónicas, aunque no se encuentra en el Daniense. Protelphidium y Rosalina tendrían sus más antiguos registros en el Maastrichtiense patagónico, sugiriendo un origen austral para estos géneros, tal como se ha señalado anteriormente para Elphidium y Cribrorotalia, cuyos más antiguos registros se conocen en el Paleogeno de Tierra del Fuego.

Las diferencias entre las asociaciones de la Patagonia septentrional y de la Patagonia austral, se deben a un diferente escenario de cuencas, al aislamiento y condiciones tendentes a la hipersalinidad en la primera, y condi- ciones de turbidez e hiposalinidad en la segunda. Una barrera, que puede haber alcanzado una gran extensión, habría separado ambos conjuntos. Sin embargo, una asociación maastrichtiense de carácter austral reconocida en la cuenca del Colorado costa afuera, sugiere que su distribución en aguas profundas alcanza al menos la latitud de Patagonia septentrional. La distribución de los taxones australes parece haber sido mayor - al menos en el Océano Atlántico - para tiempos premaastrichtienses, permitiendo su ingreso y una mayor distribución en cuencas de diferentes latitudes.

Las diferencias en la distribución de los depósitos marinos maastrichtienses respecto a los danienses en Patagonia septentrional, sugieren un leve basculamiento del inundado macizo de Somún Curá, con la elevación en el Oeste, en el área andina y el comienzo del retiro de aguas; y hundimiento en el Este, en las cuencas de Península de Valdés y del Golfo de San Jorge. El basculamiento se habría producido entre el Maastrichtiense cuspidal y el Daniense basal.

\section{SISTEMÁTICA}

El material que se describe a continuación está depositado en la Colección de foraminíferos del Laboratorio de Micropaleontología del Servicio Geológico Minero Argentino (SEGEMAR), Buenos Aires.

\section{Género Rosalina d'Orbigny, 1826}

\section{Rosalina patagonica $\mathrm{n}$. sp. Figs. 5.a-c}

Diagnosis: Una Rosalina con el lado espiral muy gruesamente perforado y con la superficie rugosa.

A Rosalina with the spiral side very coarsely perforate and with wrinkled surface.

Derivatio nominis: Patagónica, natural de la Patagonia.

Holotipo: Figura 5.c. SEGEMAR 2833.

Paratipos: Figuras 5.a y 5.b. SEGEMAR 2831-2832.

Material: 50 ejemplares.

Localidad tipo: Liu Malal, Formación Jagüel.

Edad: Maastrichtiense.

Descripción: Concha trocoespiralada, planoconvexa, lado espiral parcialmente evoluto, lado umbilical involuto, contorno levemente lobulado, periferia subredondeada. Cámaras más anchas que altas, seis a siete, raramente ocho, en la última vuelta. Lado espiral convexo; suturas limbadas e imperforadas, sobreelevadas en la parte inicial de la espira, suturas intercamerales curvadas, deprimidas las últimas tres o cuatro. Lado umbilical con suturas radiales, casi rectas a levemente curvadas, generalmente deprimidas; ombligo amplio y abierto. Pared calcárea, muy gruesamente perforada del lado espiral y en la región extra umbilical del lado umbilical; en las áreas perforadas y en especial del lado espiral la superficie es 
rugosa, mientras que es lisa en la zona umbilical no perforada. Abertura en forma de arco bajo interiomarginal, cercana a la periferia, con un pequeño labio que se continúa hacia el ombligo en una amplia extensión cameral imperforada, aberturas secundarias suturales en el margen posterior de las cámaras, las aberturas secundarias de la última vuelta permanecen abiertas.

Comentarios: La variabilidad morfológica se manifiesta en la altura de la espira, suavemente convexa a muy convexa; incluso se encontró un ejemplar con el lado espiral plano y el umbilical convexo. El desarrollo de la abertura secundaria sobre el margen posterior de las cámaras es variable, así como la amplitud del ombligo. Rosalina patagonica $\mathrm{n}$. sp. difiere de $R$. brotzeni Hofker, 1961, del Paleoceno sueco, por el mayor número de cámaras en la última vuelta, la pared más gruesamente perforada, y el lado espiral generalmente menos elevado. Rosalina elegans Hansen, 1970, del Daniense de Groenlandia, difiere por la sutura espiral deprimida, las suturas ventrales curvadas, y la pared no gruesamente perforada.

\section{Género Tehuelchella n. gen.}

Especie tipo: Tehuelchella caminosi Náñez \& Malumián n. sp.

Derivatio nominis: Tehuelche, indígena de gran talla (patagón) que da nombre a la región austral de América del Sur. Diminutivo, femenino.

Diagnosis: Concha libre, discoidal, trocoespiralada muy baja, periferia redondeada, 9 a 12 cámaras en la última vuelta, pared calcárea finamente perforada, lado espiral muy liso con suturas inconspicuas, al ras. Lado umbilical con un área umbilical grande, la cual está cubierta por una densa tuberculación que forma una figura estelar.

Test free, discoidal, very low trochospirally coiled, periphery rounded, 9 to 12 chambers in the last whorl, wall calcareous finely perforate, spiral side very smooth with inconspicuous sutures, flush. Umbilical side with a rather large umbilical area, which is covered by a dense tuberculation forming a star shape figure.

Comentarios: Es un género que en líneas generales recuerda al género Buccella del que se diferencia por su periferia redondeada, carente de carena. Aparentemente se restringe al Maastrichtiense de Patagonia septentrional, y se desconoce en el Daniense y Eoceno de Patagonia. Especies que son muy próximas y posiblemente asignables al género Tehuelchella tienen una gran distribución en la Patagonia y han sido agrupadas bajo la denominación de "Buccella" desde 1982 (Malumián, 1982), y se distribuyen ampliamente en la Patagonia desde el Oligoceno al Mioceno medio. En este grupo podría incluirse también Trochoelphidiella uniforamina D'Agostino, 1986, descrita para el Oligomioceno de Antártida (Leckie \& Webb, 1985), transferida a Ammoelphidiella Conato \& Segre, 1974 (ver
Strong \& Webb, 2000) pero que carece de las estructuras retrales que caracterizan a este último género.

\section{Tehuelchella caminosi n. gen. n. sp. Figs. 3, 4.a-f}

1995. "Pararotalia” sp., Malumián, Caramés \& Náñez, 175, 176. 2001. "Pararotalia” sp., Náñez en Caminos, 38, 39.

2001. "Pararotalia” sp., Náñez en Hugo \& Leanza, 14.

Diagnosis: Conchilla de tamaño mediano, hasta $0,30 \mathrm{~mm}$ de diámetro, con enroscamiento trocoespiralado bajo; lado espiral con todas las cámaras visibles y suturas oblicuas; lado umbilical con generalmente 9 a 12 cámaras en la última vuelta que aumentan gradualmente de tamaño, visibles alrededor del amplio ombligo circular, suturas oblicuas, levemente curvadas. Abertura no visible. Ombligo, suturas ventrales cerca del ombligo y la parte inferior de la cara apertural cubiertos por tubérculos. La tuberculación se continúa desde el ombligo hacia las suturas levemente deprimidas, dando una forma estrellada turbinada; la base de la cara apertural está cubierta por tubérculos restringidos a un área deprimida en forma de semiarco. Pared calcárea finamente perforada.

Test of medium size, up to $0.30 \mathrm{~mm}$ in diameter, enrolled in a low trochospiral coil, all chambers visible and sutures oblique on spiral side, generally 9 to 12 gradually enlarging chambers in the final whorl, visible around the broad circular umbilicus on the flat umbilical side where the sutures are oblique, gently curved. Aperture not visible. Umbilicus, ventral sutures near the umbilicus, and the lower part of the apertural face covered by tubercles. The tuberculation is continuous from the umbilicus to the slightly depressed sutures, showing a turbinate star shape; the base of the apertural face is covered by tubercles restricted to a depressed area of semiarc shape. Wall calcareous finely perforate.

Derivatio nominis: En homenaje póstumo al Dr. Roberto Caminos, insigne geólogo del Servicio Geológico Argentino y quien fue el responsable de la colecta de muestras de Laguna del Indio Muerto, con gran riqueza de ejemplares de la especie aquí descrita.

Holotipo: Figura 4.b. SEGEMAR 2826.

Paratipos: Figuras 4.a y 4.c-f. SEGEMAR 2825 y 2827 a 2830. Material: más de 6.000 ejemplares.

Localidad tipo: Laguna del Indio Muerto, Miembro Aguada Cecilio.

Edad: Maastrichtiense.

Distribución: Miembro Aguada Cecilio, Formación Arroyo Barbudo, localidades Laguna del Indio Muerto y Aguada Cecilio, Provincia de Río Negro; Formación Jagüel, Bajo de Santa Rosa (Provincia de Río Negro) y Liu Malal (Provincia de Mendoza); Formación Roca, Malargüe (provincia de Mendoza). La especie es muy abundante en la región de Laguna del Indio Muerto, Bajo de Santa Rosa y Aguada Cecilio. Es rara en Liu Malal, y presente en las muy pobres asociaciones de la región de Malargüe. 
Descripción: Concha trocoespiralada muy baja con una y media a dos y media vueltas y 17 a 22 cámaras, y con 6 y media a 12 cámaras en la última vuelta que aumentan lentamente de tamaño, contorno subcircular, periferia redondeada. Lado espiral muy levemente convexo, generalmente aplanado en la parte inicial, liso, suturas intercamerales al ras, y variablemente curvadas, sutura espiral muy levemente deprimida. Lado umbilical levemente deprimido en su parte central; el área umbilical, la base de las cámaras y las partes bajas de las suturas cubiertas por tubérculos formando una figura estelar turbinada, los tubérculos disminuyen gradualmente de tamaño desde el centro a la periferia. Abertura no visible; en la base de la cara apertural, una depresión semicircular está cubierta por tubérculos.

Comentarios: La mayoría de los ejemplares tienen un lado espiral plano a levemente convexo, pero algunos alcanzan mayor convexidad (ej. figura 4.f3). En un ejemplar de la localidad de Liu Malal, de relativa buena conservación, y en ejemplares de Aguada Cecilio se observan suturas hendidas, bordeadas por tubérculos. El foramen sería un arco interiomarginal cercano a la región umbilical.

\section{Protelphidium primitivum $\mathrm{n}$. $\mathrm{sp}$.} Figs. 5.d; 6.a-b

1995. Fissoelphidium? sp. Malumián et al., 175, 176.

Diagnosis: Un Protelphidium con suturas muy profundamente hendidas con cortas ramificaciones e incipientes estructuras pontiformes.

A Protelphidium with very deeply incised sutures with ramifications and incipient bridge like structures.

Derivatio nominis: Por ser al momento la especie más antigua del género se supone primitiva.

Holotipo: Figura 5.d. SEGEMAR 2834.

Paratipos: Figuras 6.a-b. SEGEMAR 2835-2836.

Localidad tipo: Liu Malal.

Material: Liu Malal, Formación Jagüel, 20 ejemplares; Aguada Cecilio, Miembro Aguada Cecilio, 30 ejemplares; Malargüe, Formación Roca, 30 ejemplares.

Edad: Maastrichtiense.

Descripción: Concha planoespiralada, involuta, con nueve cámaras en la última vuelta, las suturas levemente curvadas, muy hundidas formando profundos canales que en la zona extraumbilical están bordeadas por tubérculos imperforados que le dan aspecto ramificado, los tubérculos en pocos casos alcanzan a fusionarse formando estructuras ponticulares; hacia el ombligo y en las primeras suturas de la última vuelta los canales son cubiertos por material calcáreo secundario convirtiéndose en conductos. La abertura primaria no se ve; foramen interiomarginal ecuatorial, en forma de arco bajo.

Comentarios: La descripción corresponde al holotipo. El número de cámaras en la última vuelta varía entre 8 y 13, generalmente 10 a 11 . La región umbilical puede ser aplanada a deprimida, como en la mayoría de los ejem- plares de la localidad de Aguada Cecilio, o levemente convexa, con coalescencia de los tubérculos formando un umbón, como en la mayoría de los ejemplares de la región de Malargüe. Un ejemplar de esta última localidad muestra fosetas, aunque la mala preservación impidió su ilustración con microscopio electrónico de barrido. Varios ejemplares de la localidad de Aguada Cecilio son levemente asimétricos.

La especie de Protelphidium más próxima a P. primitivum, tanto morfológica como geográfica y cronológicamente, es Protelphidium hofkeri Haynes, 1956, que ha sido ilustrada varias veces para el Daniense de Patagonia septentrional y cuenca del Golfo (ej. Malumián \& Caramés, 1995). En la ilustración mediante SEM de estos últimos autores, muestra suturas deprimidas, carentes de tubérculos en las formas danienses de Patagonia, y con excelentes fotos en Hansen \& Lykke-Andersen (1976), quienes lo consideran como Nonion Montfort, 1808, puntualizando que $P$. hofkeri no tiene espacios interloculares (suturas profundamente hundidas).

Protelphidium primitivum fue asignado provisionalmente con dudas al género Fissoelphidium Smout, 1955, considerando la semejanza morfológica general y la edad Maastrichtiense de este último (Malumián et al., 1995), pero su pequeño tamaño y las características aperturales lo alejan de este género monoespecífico.

\section{AGRADECIMIENTOS}

El presente trabajo es un aporte de los Proyectos "Cenozoico de la Plataforma Continental Argentina y Patagonia, PICT 12257" y Picto "Bioestratigrafía y sedimentología del Santoniense-Maastrichtiense de la cuenca James Ross, Antártida. Acontecimientos paleobiológicos y paleoambientales", ambos de la Agencia Nacional de Promoción Científica y Tecnológica de Argentina, y del Proyecto Consolider CGL2007-63724/BTE del Ministerio de Educación y Ciencia de España.

Se agradece a los árbitros Laia Alegret y E.B. Olivero por sus observaciones y comentarios, que contribuyeron a mejorar el manuscrito. Al Servicio Geológico Minero Argentino, por la autorización de publicación.

\section{BIBLIOGRAFÍA}

Baldoni, A.M. \& Askin, R.A. 1993. Palynology of the lower Lefipán Formation (Upper Cretaceous) of Barranca de los Perros, Chubut Province, Argentina - Part II. Angiosperm pollen and discussion. Palynology, 17, 241-264.

Barcat, C., Cortiñas, J.S., Nevistic, V.A. \& Zucchi, H.E. 1989. Cuenca Golfo San Jorge. In: Cuencas Sedimentarias Argentinas (Eds. G. Chebli \& L. Spalletti). Serie Correlación Geológica, Universidad Nacional de Tucumán, 6, 319-345. 
Barker, F.F., Dalziel, I.W.D., Elliott, D.H., Von der Borch, C.C., Thompson, R.W., Plfker, G., Tjalsma, R.C., Wise, S.W., Dinkelman, M.G., Gombos, A.M., Lonardi, A., Tarney, J., Harris, W. \& Sliter, W.V. 1977. Evolution of the Southwestern Atlantic Ocean Basin: Results of Leg 36, DSDP. In: Barker P.F., Dalziel, I.W.D. et al. Initial Reports of the Deep Sea Drilling Project, 36, 993-1014.

Barrio, C.A. 1990a. Paleogeographic control of Upper Cretaceous tidal deposits, Neuquén Basin, Argentina. Journal of South American Earth Sciences, 3, 31-49.

Barrio, C.A. 1990b. Late Cretaceous-Early Tertiary sedimentation in a semi-arid foreland basin (Neuquén Basin, western Argentina). Sedimentary Geology, 66, 255-275.

Barron, E.J. 1987. Global Cretaceous paleogeographyInternational Geologic Correlation Program Project 191. Palaeogeography, Palaeoclimatology, Palaeoecology, 59, 207-216.

Basov, I.A. \& Krasheninnikov, V.A. 1983. Benthic foraminifers in Mesozoic and Cenozoic sediments of the southwestern Atlantic as an indicator of paleoenviroment. DSDP Leg 71. In: W.J. Ludwig, V.A. Krasheninnikov et al. Initial Reports of the Deep Sea Drilling Project, 71, 739-787.

Beckmann, J.P. 1978. Late Cretaceous smaller benthic foraminifers from sites 363 and 364 DSDP Leg 40, Southeast Atlantic Ocean. Initial Reports of the Deep Sea Drilling Project, 40, 759-781.

Bertels, A. 1970. Hiltermannia n. gen. (Foraminiferida) del Cretácico superior (Maastrichtiano) de Argentina. Ameghiniana, 7, 167-172.

Bertels, A. 1972. Buliminacea y Cassidulinacea (Foraminiferida) guías del Cretácico Superior (Maastrichtiano medio) y Terciario inferior (Daniano inferior) de la República Argentina. Revista Española de Micropaleontología, 4, 127-253.

Bertels, A. 1974. Upper Cretaceous (middle Maastrichtian) ostracodes of Argentina. Micropaleontology, 21, 97-130.

Bertels, A. 1975. Ostracode ecology during the Upper Cretaceous and Cenozoic in Argentina. Bulletin American Paleontologist, 65, 282, 317-351.

Bertels, A. 1978. Microfauna del Cretácico superior y del Terciario inferior. VII Congreso Geológico Argentino, Relatorio, 163-175.

Bertels, A. 1979. Paleobiogeografía de los foraminíferos del Cretácico superior y Cenozoico de América del Sur. Ameghiniana, 16, 273-356.

Bertels, A. 1980. Estratigrafía y foraminíferos (Protozoa) bentónicos del límite Cretácico-Terciario en el área tipo de la Formación Jagüel, Provincia de Neuquén, República Argentina. II Congreso Argentino de Paleontología y Bioestratigrafía y I Congreso Latinoamericano de Paleontología, Actas, 2, 47-91.

Bertels, A. 1988. Cretaceous Foraminifera of Argentina: Biogeographic tendencies. Revista Brasileira Geociências, 18, 299-311.

Bertels, A. 1995. The Cretaceous-Tertiary boundary in Argentina and its ostracodes. In: Ostracoda and Biostratigraphy (ed. J. Riha). Balkema, Rotterdam, 163-170.
Bettenstaedt, F. \& Wicher, C. 1955. Stratigraphic correlation of Upper Cretaceous and Lower Cretaceous in the Tethys and Boreal by aid of microfossils. Fourth World Petroleum Congress, Proc. Sect. 1/D, 5, 493-516.

Brito, P.M. \& Suárez, M.E. 2003. Late Cretaceous Belonostomus (Pisces, Actinopterygii, Aspidorhynchidae) from Algarrobo, Chile, with comments on aspidorhynchid paleodistribution in South America. Revista Geológica de Chile, 30, 117-127.

Boltovskoy, E. 1976. Distribution of Recent foraminifera of the South American Region. In: Foraminifera, 2, (Eds. R.H. Hedley \& C.G. Adams) Academic Press, London, 171-236.

Camacho, H.H. 1967. Las transgresiones del Cretácico Superior y Terciario de la Argentina. Revista de la Asociación Geológica Argentina, 22, 253-280.

Caminos, R. 2001. Hoja Geológica n 4166-IV, Valcheta, Provincia de Río Negro. Servicio Geológico Minero Argentino, Boletín, 310, 1-73.

Caramés, A. 1993. Foraminíferos y bioestratigrafía del Cretácico Superior y Terciario Inferior del Sur de Argentina. Tesis Doctoral. Universidad de Buenos Aires, tomo 1, 208 pp., tomo 2, $178 \mathrm{pp}$. (inédito).

Caramés, A. \& Malumián, N. 2006. La Familia Rzehakinidae (Foraminifera) en el Cretácico superior-Paleogeno de la cuenca Austral y la plataforma continental atlántica adyacente, Argentina. Ameghiniana, 43, 649-668.

Caramés, A., Malumián, N. \& Náñez, C. 2004. Foraminíferos del Paleógeno del Pozo Península Valdés (PV.es-1), Patagonia septentrional, Argentina. Ameghiniana, 41, 461-474.

Casadío, S. 1998. Las ostras del límite Cretácico-Paleógeno de la cuenca Neuquina (Argentina). Su importancia bioestratigráfica y paleobiogeográfica. Ameghiniana, 35, 449-472.

Casadío, S., Rodríguez, M.F., Reichler, V.A. \& Camacho, H.H. 1999. Tertiary nautiloids from Patagonia, southern Argentina. Ameghiniana, 36, 182-202.

Casamiquela, R.M. 1978. La zona litoral de la trasgresión Maästrichtense en el norte de la Patagonia. Aspectos ecológicos. Ameghiniana, 15, 137-148.

Cecioni, G. 1970. Esquema de paleogeografía chilena. Editorial Universitaria, Santiago de Chile, $141 \mathrm{pp}$.

Ciesielski, P.F. \& Wise, W. 1977. Geologic history of the Maurice Ewing bank of the Falkland Plateau (Southwest Atlantic sector of the Southern Ocean) based on piston and drill cores. Marine Geology, 25, 175-207.

Ciesielski, P.F., Sliter, W.V., Wind, F.H. \& Wise, S. 1977. Paleoenvironmental analysis and correlation of a Cretaceous Islas Orcadas core from the Falkland (Southwest Atlantic sector of the Southern Ocean) based on piston and drill cores. Marine Micropaleontology, 2, 27-34.

Concheyro, A. \& Villa, G. 1996. Maestrichtian-Danian (K/P) calcareous nannofossils in the Liu Malal section, Northern Patagonia, Argentina. Palaeopelagos, 6, 281-297.

Cortés, J.M. 1987. Descripción Geológica de la Hoja 42h, "Puerto Lobos". Provincia del Chubut. Dirección Nacional de Minería y Geología, Boletín, 202, 1-94. 
Crame, J.A., McArthur, J.M., Pirrie, D. \& Riding, J.B. 1999. Strontium isotope correlation of the basal Maastrichtian Stage in Antarctica to the European and US biostratigraphic schemes. Journal of the Geological Society of London, 156, 957-964.

Dalziel, I.W.D. \& Elliot, D.H., 1971. Evolution of the Scotia arc. Nature, 233, 246-252.

DeVargas, C., Norris, R., Zaninetti, L., Gibb, S.W. \& Pawlowski, J. 1999. Molecular evidence of cryptic speciation in planktonic foraminfers and their relation to oceanic provinces. Proceedings of the National Academy of Sciences USA, 96, 2864-2868.

Dingle, R. 1988. Marine Ostracod Distributions during the Early Breakup of Southern Gondwanaland. In: Evolutionary Biology on Ostracoda (Ed. T. Hanai, N. Ikeya \& K. Ishizaki). Elsevier. Developments in palaeontology and stratigraphy, 11, 841-854.

El-Shinnawi, M.A. 1972. Some Maestrichtian new species of Gabonella (Forams) from Hurgada well N ${ }^{\circ} 134$, Eastern Desert, Egypt. Revue de Micropaléontologie, 14, 235-247.

Fahrni, J. \& Pawlowski , J. 1995. Identification of actins in foraminifera: Phylogenetic implications. European Journal of Protistology, 31, 161-166.

Feldmann, R.M. \& Schweitzer, C.E. 2006. Paleogeography of Southern Hemisphere decapod Crustacea. Journal of Paleontology, 80, 83-103.

Feldmann, R.M., Casadío, S., Chirino-Gálvez, L. \& AguirreUrreta, M., 1995. Fossil decapod crustaceans from the Jagüel and Roca Formations (Maastrichtian-Danian) of the Neuquén Basin, Argentina. The Paleontological Society Memoir, 43, 1-22.

Fleming, C.A. 1962. New Zealand biogeography: a paleontologist's approach. Tuatara, 10, 53-108.

Galeazzi, J.S. 1996. Cuenca Malvinas. In: Geología y Recursos Naturales de la plataforma continental Argentina, Relatorio del XIII Congreso Geológico Argentino y III Congreso de Exploración de Hidrocarburos (Eds. V.A. Ramos \& M.A. Turic), 5, 273-309.

Galeazzi, J.S.1998. Structural and Stratigraphic Evolution of the Western Malvinas Basin, Argentina. American Association Petroleum Geologist, Bulletin, 82, 596-636.

Gasparini, Z., Salgado, L. \& Parras, A. 2007. Late Cretaceous plesiosaurs from northern Patagonia, Argentina. Geological Journal, 42, 185-202.

Griffin, M. \& Hünicken, M.A. 1994. Late Cretaceous-Early Tertiary gastropods from Southwestern Patagonia, Argentina. Journal of Paleontology, 68, 257-274.

Griffin, M., Casadío, S . \& Parras, A. 2005. Maastrichtian and Danian species of Turkostreini (Ostreidae, Crassostreinae) from the Neuquén Basin, Argentina. Ameghiniana, 42, 257-276.

Groeber, P. 1959. Supracretácico. In: Geografía de la República Argentina. Sociedad Argentina de Estudios Geográficos GAEA, 2, 1-165.

Groeber, P. 1963. La Cordillera entre las latitudes $22^{\circ} 20^{\prime} \mathrm{y}$ $40^{\circ}$. Academia Nacional de Ciencias, 43, 111-176.

Guerstein, G.R., Prámparo, M.C., Junciel, G.L., Guler, M.V.
\& Daners, G. 2004. Bioestratigrafía basada en dinoflagelados del Maastrichtiano-Daniano del Atlántico sudoccidental. X Reunión Argentina de Sedimentología, Acta de Resúmenes, 190-192.

Hansen, H.J. \& Lykke-Andersen, A-L. 1976. Wall structure and classification of fossil and recent elphidiid and nonionid Foraminifera. Fossils and Strata, 10, 1-37.

Henderson, R.A. \& McNamara, K.J. 1985. Taphonomy and ichnology of cephalopod shells in a Maastrichtian chalk from Western Australia. Lethaia, 18, 305-322.

Huber, B.T. 1984. Late Cretaceous foraminiferal biostratigraphy, paleoecology and paleobiogeography of the James Ross Island region, Antarctic Peninsula. M.Sc. thesis, The Ohio State University, 246 pp. (inédito).

Huber, B.T. 1988. Upper Campanian-Paleocene foraminifera from the James Ross Island region, Antarctic Peninsula. In: Geology and Paleontology of Seymour Island, Antarctica (Eds. R.M Feldmann \& M.O. Woodburne). Geological Society of America, Memoir, 169, 163-252.

Huber, B.T. 1992a. Paleobiogeography of CampanianMaastrichtian foraminifera in the southern high latitudes. Palaeogeography, Palaeoclimatology, Palaeoecology, 92, 325-360.

Huber, B.T. 1992b. Upper Cretaceous planktic foraminiferal biozonation for the Austral Realm. Marine Micropaleontology, 20, 107-128.

Huber, B.T. 1994. Ontogenetic morphometrics of some Late Cretaceous trochospiral planktonic foraminifera from the Austral realm. Smithsonian Contributions to Paleobiology, 77, 1-85.

Huber, B.T. \& Watkins, D.K. 1992. Biogeography of Campanian-Maastrichtian calcareous plankton in the region of the Southern Ocean: Paleogeographic and paleoclimatic implications. The Antarctic paleoenvironment: A perspective on global change. Antarctic Research Series, 56, 31-60.

Huber, B.T. \& Webb, P.N. 1986. Distribution of Frondicularia rakauroana (Finlay) in the southern high latitudes. Journal of Foraminiferal Research, 16, 135-140.

Hugo, C.A. \& Leanza, H.A. 2001. Hoja Geológica 3966-III, Villa Regina, Provincia de Río Negro. Servicio Geológico Minero Argentino, Boletín, 309, 1-53.

Jannou, G. 2007. Radiolarios del Paleógeno de la cuenca Austral, Tierra del Fuego, Argentina. Ameghiniana, 44, 447-466.

Kaiho, K. 1998. Phylogeny of deep-sea calcareous trochospiral benthic foraminifera: evolution and diversification. Micropaleontology, 44, 291-311.

Kauffman, E.G., 1973. Cretaceous Bivalvia. In: Atlas of Paleogeography (Ed. A. Hallam ). Elsevier, 351-383.

Kielbowicz, A. 1980. Estudio de la microfauna del Cretácico superior en la zona de Lago Pellegrini, Provincia de Río Negro. Trabajo Final de Licenciatura, Universidad de Buenos Aires, 77 pp. (inédito).

Koutsoukos, E.A. 1992. Late Aptian to Maastrichtian foraminiferal biogeography and palaeoceanography of the Sergipe Basin, Brazil. Palaeogeography, Palaeoclimatology, Palaeoecology, 92, 295-324.

Koutsoukos, E.A.M. \& Klasz, I. de 2000. Late Cretaceous 
foraminiferal biogeography (Families Bolivinidae, Buliminellidae, Gavelinellidae, Siphogenerinoididae, Turrilinidae) in northeastern Brazilian shelf and central West African basins. Cretaceous Research, 21, 381-405.

Krasheninnikov, V.A. \& Basov, I.A. 1986. Late Mesozoic and Cenozoic stratigraphy and geological history of the South Atlantic high latitudes. Palaeogeography, Palaeoclimatology, Palaeoecology, 55, 145-148.

Lawver, L.A., Gahagan, L.M. \& Coffin, M.F. 1992. The development of paleoseaways around Antarctica. In: The Antarctic Paleoenvironment: A perspective on global change. Antarctic Research Series, 56, 7-30.

Leckie, R.M. \& Webb, P.-N. 1985. Late Paleogene and early Neogene Foraminifers of Deep Sea Drilling Project Site 270, Ross Sea, Antarctica. In: Kennett, J.P., von der Borch, C.C. et al., Initial Reports of the Deep Sea Drilling Project, 90, 1093-1141.

Lizuaín, A.1983. Descripción Geológica de la Hoja 38j, Salinas del Gualicho, Provincia de Río Negro. Servicio Geológico Nacional, Boletín, 195, 48 pp.

Lutze, G.F. \& Thiel, H. 1989. Epibenthic foraminifera from elevated microhabitats: Cibicides wuellerstorfi and Planulina ariminensis. Journal of Foraminiferal Research, 19, 153-158.

Macellari, C.E. 1985. Paleogeografía y edad de la fauna de Maorites-Gunnarites (Ammonoidea) del Cretácico superior de la Antártida y Patagonia. Ameghiniana, 21, 223-242.

Macellari, C.E. 1988. Cretaceous paleogeography and depositional cycles of western South America. Journal of South American Earth Sciences, 1, 373-418.

Malumián, N. 1968. Foraminíferos del Cretácico Superior y Terciario del subsuelo de la Provincia Santa Cruz, Argentina. Ameghiniana, 5, 191-227.

Malumián, N. 1979. Aspectos paleoecológicos de los foraminíferos del Cretácico de la cuenca Austral. Ameghiniana, 15, 149-160.

Malumián, N. 1982. Foraminíferos bentónicos de la Formación Carmen Silva, Mioceno, Isla Grande de Tierra del Fuego. Ameghiniana, 19, 37-66.

Malumián, N. 1990. Foraminíferos de la Formación Man Aike (Eoceno, sureste del Lago Cardiel), Provincia de Santa Cruz. Revista de la Asociación Geológica Argentina, 45, 365-385.

Malumián, N. 1994. Foraminíferos nuevos o característicos del Eoceno medio de cuenca Austral: significado paleozoogeográfico. Ameghiniana, 31, 139-151.

Malumián, N. 1999. La sedimentación en la Patagonia Extraandina. In: Geología Argentina (Ed. R. Caminos). Instituto de Geología y Recursos Minerales, Anales, 29, 557-612

Malumián, N. \& Báez, A.M. 1976. Outline of Cretaceous stratigraphy of Argentina, In: Evenements de la partie moyenne du Cretace. Annales du Museum d'Histoire Naturelle de Nice, 4, XXVII, 1-10.

Malumián, N. \& Caramés, A. 1995. El Daniano marino de Patagonia (Argentina): Paleobiogeografía de los foraminíferos bentónicos. In: Paleogeno de América del Sur (Ed. C. Náñez). Asociación Paleontológica Argentina, Publicación Especial, 3, 83-105.
Malumián, N. \& Caramés, A. 2002. Foraminíferos de sedimentitas ricas en carbono orgánico: Formación La Barca, Paleoceno superior, Tierra del Fuego, Rep. Argentina. Revista de la Asociación Geológica Argentina, 57, 219-231.

Malumián, N. \& Masiuk, V. 1973. Asociaciones foraminiferológicas fósiles de la República Argentina. V Congreso Geológico Argentino, Actas, 3, 435-453.

Malumián, N. \& Náñez, C. 1984. Foraminíferos. IX Congreso Geológico Argentino, Relatorio, 2-7, 486-497.

Malumián, N. \& Náñez, C. 1990. Foraminíferos aglutinados del Cretácico Superior de cuenca Austral (Provincia de Santa Cruz), Argentina. In: Bioestratigrafía de los Sistemas regionales del Jurásico y Cretácico de América del Sur (Ed. W. Volkheimer). Comité Sudamericano del Jurásico y Cretácico, Mendoza, 2, 497-551.

Malumián, N. \& Náñez, C. 1996. Microfósiles y Nanofósiles Calcáreos de la Plataforma Continental. In: Geología y Recursos Naturales de la Plataforma Continental Argentina, Relatorio del XIII Congreso Geológico Argentino y III Congreso de Exploración de Hidrocarburos (Eds. V.A. Ramos \& M.A. Turic), 5, 73-93.

Malumián, N. \& Náñez, C. 2002a. Foraminíferos de la transgresión maastrichtiana en la Provincia de Santa Cruz, República Argentina. XV Congreso Geológico Argentino, Actas, 518-522.

Malumián, N. \& Náñez, C. 2002b. Los foraminíferos: su significado geológico y paleoambiental. Geología y recursos naturales de Santa Cruz. XV Congreso Geológico Argentino, Relatorio, 481-494.

Malumián, N. \& Panza, J.L. 2000. Hoja Geológica 5172-III. Yacimiento Río Turbio, Provincia de Santa Cruz. Servicio Geológico Minero Argentino, Boletín, 247, 1-108.

Malumián, N., Nullo, F.E. \& Ramos, V.A. 1983. The Cretaceous of Argentina, Chile, Paraguay and Uruguay. In: The Phanerozoic Geology of the World II, The Mesozoic, B. (Eds. M. Moullade \& A.E.M. Nairn). Elsevier, The Netherlands, 9, 265-304.

Malumián, N., Náñez, C. \& Caramés, A.A. 1991. Unilocular foraminifera of reticular surface from Argentina. Micropaleontology, 37, 393-406.

Malumián, N., Caramés, A. \& Náñez, C. 1995. El cambio en los foraminíferos bentónicos en el pasaje CretácicoPaleogeno, cuencas Neuquina y del Colorado, Argentina. $6^{\circ}$ Congreso Argentino de Paleontología y Bioestratigrafía, Actas, 173-178.

Malumián, N., López Cabrera, M.I., Náñez, C. \& Olivero, E. 2007. Bioerosion patterns in Cretaceous-Cenozoic benthic foraminiferal tests from Patagonia and Tierra del Fuego Island, Argentina. In: Sediments-Organism Interactions; a multifaceted Ichnology (Eds. Bromley et al.) SEPM Special Publication, 88, 299-306.

Marengo, H.G., Echeverría, A. \& Concheyro, A. 2005. Microfósiles marinos miocenos del subsuelo de la ciudad de Buenos Aires. XVI Congreso Geológico Argentino, Actas, 317-324.

Markwick, P.J. \& Valdes, P.J. 2004. Palaeo-digital elevation models for use as boundary conditions in couple oceanatmosphere GCM experiments: a Maastrichtian (Late Cre- 
taceous) example. Palaeogeography, Palaeoclimatology, Palaeoecology, 213, 37-63.

Masiuk, V. 1967. Estratigrafía del Rocanense del Puesto P. Álvarez. Curso inferior del Río Chico, Prov. del Chubut. Revista del Museo de la Plata (n.s.) Sección Paleontología, 5, 197-258.

Masiuk, V., Becker, D. \& García Espiasse, A. 1976. Micropaleontología y sedimentología del pozo YPF Ch.PVes-1 (Península Valdés) Provincia del Chubut, República Argentina. ARPEL XXIV. Reunión a nivel de expertos. Buenos Aires, 28 pp.

Medina, F.A. \& Olivero, E. 1994. Paleontología de la Formación Lefipán (Cretácico-Terciario) en el valle medio del río Chubut. Revista de la Asociación Geológica Argentina, 48, 104.

McGowran, B. 1978. Early Tertiary foraminiferal biostratigraphy in Southern Australia: a progress report. In: The Crespin Volume: Essay in honour of Irene Crespin (eds. D.J. Belford \& V. Scheibnerová). Department of National Development, Bureau of Mineral Resources, Geology and Geophysics, Bulletin, 192, 83-95.

Muñoz R., C. \& Palma-Heldt, S. 2006. Registro de Eslamobranquios en la Formación Quiriquina, Cretácico Superior. VIII Región, Chile. Ameghiniana, Suplemento Resúmenes, 43, 47R.

Murray, J. W. 1976. A method of determining proximity of marginal seas to an ocean. Marine Geology, 22, 103-119.

Musacchio, E. 1995. Estratigrafía y micropaleontología del Jurásico y el Cretácico en la comarca del valle medio del río Chubut, Argentina. VI Congreso Argentino de Paleontología y Bioestratigrafía, Actas, 179-187.

Musacchio, E. 2000. Biostratigraphy and biogeography of Cretaceous charophytes from South America. Cretaceous Research, 21, 211-220.

Náñez, C. 1998. Informe micropaleontológico sobre muestras de las Hojas Las Plumas 4366-3, provincia del Chubut, Bosque Petrificado 4969-4 y Destacamento La María, provincia de Santa Cruz. Servicio Geológico Minero Argentino, IGRM (inédito).

Náñez, C. \& Concheyro, A. 1997. Límite Cretácico-Paleógeno. In: Geología y Recursos Minerales del Departamento Añelo, Provincia del Neuquén, República Argentina (Eds. A. Ardolino \& M. Franchi). Dirección Nacional del Servicio Geológico, Anales, 25, 129-149.

Náñez, C. \& Malumián, N. 2004. Distribution of conspicuous or endemic benthic foraminifera across the K/P boundary in Patagonia. X Reunión Argentina de Sedimentología, Acta de Resúmenes, 193-195.

Natland, M.L., González, E.P., Cañón, A. \& Ernst, M. 1974. A System of Stages for correlation of Magallanes Basin sediments. Geological Society of America Memoir, 139, 1-125.

Nederbragt, A.J. 1998. Quantitative biogeography of late Maastrichtian planktic foraminifera. Micropaleontology, 44, 385-412.

Néraudeau, D. \& Mathey, B. 2000. Biogeography and diversity of South Atlantic Cretaceous echinoids: Implications for circulation patterns. Palaeogeography, Palaeoclimatology, Palaeoecology, 156, 71-88.
Odébòde, M.O.O. 1987. The taxonomic position of the Late Cretaceous foraminiferal genus Gabonita de Klasz, Marie \& Meijer. Neues Jahrbuch für Mineralogie, Geologie und Paleontologie, Monatshefte, 1987, 146-154.

Ogg, J.G., Agterberg, F.P. \& Gradstein, F.M. 2004. The Cretaceous Period. In: A Geologic Time Scale (Eds. F.M. Gradstein, J.G. Ogg \& A.G. Smith). Cambridge University Press, 344-383.

Olivero, E.B. \& Medina, F.A. 1994. Sedimentología de la Formación Lefipán (Cretácico-Terciario) en el valle medio del Río Chubut. Revista de la Asociación Geológica Argentina, 48, 105-106.

Olivero, E.B. \& Medina, F.A. 2000. Patterns of Late Cretaceous ammonite biogeography in southern high latitudes: The Family Kossmaticeratidae in Antarctica. Cretaceous Research, 21, 269-279.

Olivero, E.B., Medina, F.A. \& Camacho, H.H. 1990. Nuevos hallazgos de moluscos con afinidades australes en la Formación Lefipán (Cretácico Superior, Chubut): Significado paleogeográfico. V Congreso Argentino de Paleontología y Bioestratigrafía, Actas, I, 129-135.

Olivero, E.B., Malumián, N., Palamarczuk, S. \& Scasso, R. 2002. El Cretácico tardío-Paleogeno del área del Río Bueno, costa atlántica de la Isla Grande de Tierra del Fuego. Asociación Geológica Argentina, Revista, 57, 199-218.

Olivero, E.B., Malumián, N. \& Palamarczuk, S. 2003. Estratigrafía del Cretácico Superior-Paleoceno del área de Bahía Thetis, Andes fueguinos, Argentina: acontecimientos tectónicos y paleobiológicos. Revista Geológica de Chile, 30, 245-263.

Page, R., Ardolino, A., de Barrio, R.E., Franchi, M., Lizuaín, A., Page, S. \& Silva Nieto, D. 1999. Estratigrafía del Jurásico y Cretácico del Macizo de Somún Curá, Provincias de Río Negro y Chubut. In: Geología Argentina (Ed. R. Caminos). Instituto de Geología y Recursos Minerales, Anales, 29, 460-488.

Palamarczuk, S. \& Olivero, E. 2000. Asociaciones de dinoflagelados del Cretácico Superior-Paleogeno en la costa atlántica de la isla Grande de Tierra del Fuego. XI Simposio Argentino de Paleobotánica y Palinología, Resúmenes, 85.

Palamarczuk, S.,Olivero,E.\& Martinioni,D. 1998. Distribución geográfica de dinoquistes maastrichtianos y danianos en Argentina centro-austral, Chile y Antártida. VII Congreso Argentino de Paleontología y Bioestratigrafía, Resúmenes, 25.

Papú, O.H. 2002. Nueva microflora de edad maastrichtiana en la localidad de Calmu-Co, sur de Mendoza, Argentina. Ameghiniana, 39, 415-426.

Parma, S.G. \& Casadío, S. 2005. Upper Cretaceous-Paleocene echinoids from northern Patagonia, Argentina. Journal of Paleontology, 79, 1072-1087.

Parras, A.M., Casadío, S. \& Pires, M. 1998. Secuencias depositacionales del grupo Malargüe y el límite CretácicoPaleógeno, en el sur de la Provincia de Mendoza, Argentina. In: Paleógeno de América del Sur y de la Península Antártica (Ed. S. Casadío). Asociación Paleontológica Argentina, Publicación Especial, 5, 61-69.

Pascual, R. 1998. The history of South American land mammals: the seminal Cretaceous-Paleocene transition. In: 
Paleógeno de América del Sur y de la Península Antártica (Ed. S. Casadío). Asociación Paleontológica Argentina, Publicación Especial, 5, 9-18.

Pires, M., Náñez, C., Casadío, S. \& Parras, A. 1999. Sedimentología, paleontología y estratigrafía del límite Cretácico-Paleógeno en Liu Malal, Mendoza. XIV Congreso Geológico Argentino, Actas, 1, 463-466.

Polanski, J. 1964. Descripción geológica de la Hoja Geológica 25a Volcán de San José, provincia de Mendoza. Dirección Nacional de Geología y Minería, Boletín, 98, 94 pp.

Prámparo, M.B. \& Papú, O.H. 2002. Palinoestratigrafía del Maastrichtiano Superior, Cerro Butaló, sur de Mendoza, Argentina. $6^{o}$ Simposio sobre o Cretáceo do Brasil y $2^{\circ}$ Simposio sobre el Cretácico de América del Sur, Boletim, 163-167.

Prámparo, M.B. \& Papú, O.H. 2003. Palinomorfos terrestres del Maastrichtiano superior en el Cerro Butaló, sur de Mendoza, Argentina. $12^{\circ}$ Simposio Argentino de Paleobotánica y Palinología. Ameghiniana Suplemento Resúmenes, 40, 14R.

Quilty, P.G. 1992. Upper Cretaceous benthic foraminifers and paleoenvironments, southern Kerguelen Plateau, Indian Ocean. Proceedings of the Ocean Drilling Program, Scientific Results, 120, 393-493.

Quilty, P.G. 2002. Paleoenvironmental Significance of Late Cretaceous Foraminifers from ODP Site 1138 Samples, a Dredge and Eltanin Core E54-7, Kerguelen Plateau, Indian Ocean. Proceedings of the Ocean Drilling Program, Scientific Results, 183, 1-28.

Ramos, V.A. 1996. Geología de las Islas Georgias del Sur. In: Geología y Recursos Naturales de la Plataforma Continental Argentina, Relatorio del XIII Congreso Geológico Argentino y III Congreso de Exploración de Hidrocarburos (Eds. V.A. Ramos \& M.A. Turic), 359-368.

Rapalini, A.E. 2007. A paleomagnetic analysis of the Patagonian Orocline. Geologica Acta, 5, 287-294.

Revets, S.A. 1996. The generic revision of five families of Rotaliine Foraminifera. Part II. The Anomalinidae, Alabaminidae, Cancrisidae and Gavelinellidae. Cushman Foundation for Foraminiferal Research Special Publication, 34, 57-113.

Revets, S.A. 2001. The Revision of Gavelinella Brotzen, 1942, Berthelina Malapris, 1965 and Lingulogavelinella Malapris, 1965. Cushman Foundation for Foraminiferal Research, Special Publication, 37, 1-110.

Riccardi, A.C. 1987. Cretaceous paleogeography of Southern South America. Palaeogeography, Palaeoclimatology, Palaeoecology, 59, 169-195.

Riccardi, A.C. 1988. The Cretaceous system of southern South America. Geological Society of America, Memoir, 168, $161 \mathrm{pp}$.

Riccardi, A.C., Damborenea, S.E., Manceñido, M.O. \& Ballent, S. 1999. El Jurásico y Cretácico de la Cordillera Principal y la cuenca Neuquina. 3 Bioestratigrafía. In: Geología Argentina (Ed. R. Caminos). Instituto de Geología y Recursos Minerales, Anales, 29, 419-432.

Romero, E.J. 1973. Polen fósil de "Nothofagus" ("Nothofagidites") del Cretácico y Paleoceno de Patagonia. Revista del Museo de La Plata, 7, 291-303.
Ronchi, D. \& Angelozzi, G. 1994. Bioestratigrafía del Cretácico-Terciario en dos pozos ubicados al oeste de la Cuenca Austral. Boletín de Informaciones Petroleras, Junio, 65-76.

Salfity, J.A. \& Zambrano, J.J. 1990. Cretácico. In: Geología de América del Sur (Eds. J.F. Bonaparte, A. Toselli \& F.G. Aceñolaza). Universidad Nacional de Tucumán, Serie Correlación Geológica 2, 3, 185-284.

Scheibnerová, V. 1971. Foraminifera and their Mesozoic biogeoprovinces. Records of the Geological Survey of New South Wales, 13, 135-174

Scheibnerová, V. 1973. Possible Cretaceous migration routes of Austral Foraminifera. Quarterly Notes of the Geological Survey of New South Wales, 12, 7-18.

Sliter, W.V. 1977. Cretaceous foraminifera from the southwest Atlantic Ocean, Leg 36, Deep Sea Drilling Project. In: P.F. Barker, I.W.D. Dalziel et al. Initial Reports of the Deep Sea Drilling Project, 36, 519-573.

Spalletti, L.A. 1996. Estuarine and shallow-marine sedimentation in the Upper Cretaceous-Lower Tertiary west-central Patagonian Basin (Argentina). In: Geology of siliciclastic shelf seas (Eds. M. Debatist \& P. Jacobs). Geological Society Special Publication, 117, 81-93.

Stevens, G.R. 1980. Southwest Pacific faunal palaeobiogeography in Mesozoic and Cenozoic times: a review. Palaeogeography, Palaeoclimatology, Palaeoecology, 31, 153-196.

Stinnesbeck, W. 1986. Faunistic and paleoecological conditions of the Quiriquina Formation (Maastrichtian) of central Chile. Palaeontographica A, 194, 99-237.

Strong, C.P. \& Webb, P. N. 2000. Oligocene and Miocene Foraminifera from CRP-2/2A, Victoria Land Basin, Antarctica. Terra Antartica, 7, 461-472.

Suárez, M.E. 2001. Fossil fish faunas from the Quiriquina Formation, Late Cretaceous (Maastrichtian) of Chile, South America. International Meeting on Mesozoic Fishes, Systematics, Paleoenvironments and Biodiversity, Abstract Book, 3, 59.

Suárez, M.E. \& Cappetta, H. 2004. Sclerorhynchid teeth (Neoselachii, Sclerorhynchidae) from the Late Cretaceous of the Quiriquina Formation, central Chile. Revista Geológica de Chile, 31, 89-103.

Suárez, M. \& Pettigrew, T.H. 1976. An upper Mesozoic island-arc-back-arc system in the southern Andes and South Georgia. Geological Magazine, 113, 305-328.

Tambareau, Y. 1982. Les ostracodes et l'histoire géologique de l'Atlantique sud au Crétacé. Centres de Recherches Exploration-Production Elf-Aquitaine, 6, 1-37.

Tinoco, I.M. 1975. Estabelecimento e desenvolvimento da Província biogeográfica das Índias Ocidentais. Arquivos do Museo Nacional, Rio de Janeiro, 55, 167-171.

Tinoco, I.M. 1978. Foraminíferos bentônicos da Formaçao Gramame (Cretáceo Superior, Maestrichtiano de Pernambuco). Anais do 30 Congresso Brasileiro de Geologia (Recife), 2, 1032-1046.

Tunik, M.A. 2003. Interpretación paleoambiental de los depósitos de la Formación Saldeño (Cretácico superior) en la alta Cordillera de Mendoza, Argentina. Revista de la Asociación Geológica Argentina, 58, 417-433. 
Uliana, M.A. \& Biddle, K.T. 1988. Mesozoic-Cenozoic paleogeographic and geodynamic evolution of southern South America. Revista Brasileira de Geociencias, 18, 172-190.

Uliana, M.A. \& Dellapé, D.A. 1981. Estratigrafía y evolución paleoambiental de la sucesión maestrichtiana-eoterciaria del engolfamiento neuquino (Patagonia septentrional). VIII Congreso Geológico Argentino, Actas, 3, 673-711.

Van Morkhoven, F.P.C.M., Berggren, W.A. \& Edwards, A.S. 1986. Cenozoic cosmopolitan deep-water benthic foraminifera. Bulletin du Centre des Recherchers ExplorationProduction Elf-Aquitaine, 11, 1-421.

Vizcaíno, S.F., Pascual, R., Reguero, M.A. \& Goin, F.J. 1998. Antarctica as background for mammalian evolution. In: Paleógeno de América del Sur y de la Península Antártica (Ed. S. Casadío). Asociación Paleontológica Argentina, Publicación Especial, 5, 199-209.

Webb, P.N. 1971. New Zealand Late Cretaceous (Haumurian) Foraminifera and Stratigraphy: A summary. N.Z. Journal of Geology and Geophysics, 14, 795-828.

Webb, P.N. 1973. Upper Cretaceous-Paleocene Foraminifera from Site 208 (Lord Howe Rise, Tasman Sea), DSDP, Leg 21. Initial Reports Deep Sea Drilling Project, 21, 541-573.
Wichmann, R. 1927. Sobre la facies lacustre senoniana de los estratos con dinosaurios y su fauna. Boletín de la Academia Nacional de Ciencias, 30, 383-405.

Widmark, J.G.V. 2002. Biogeography of terminal Cretaceous benthic foraminifera: deep-water circulation and trophic gradients in the deep South Atlantic. Cretaceous Research, 21, 367-379.

Yrigoyen, M.R. 1989. Cuenca de Malvinas. In: Cuencas Sedimentarias Argentinas (Eds. G. Chebli \& L. Spalletti). Serie Correlación Geológica 6. Universidad Nacional de Tucumán, 467-476.

Zinsmeister,W.J. 1979. Biogeographic significance of the Late Mesozoic and Early Tertiary molluscan faunas of Seymour Island (Antarctic Peninsula) to the final breakup of Gondwanaland. In: Historical Biogeography, Plate Tectonics and The Changing Environment (Eds. J. Gray, J. \& A. Boucot). Proceedings of the Thirty-seventh Annual Biology Colloquium and Selected Papers, Oregon State University Press, 349-355.

Manuscrito recibido: 19 de Mayo, 2008 Manuscrito aceptado: 22 de Septiembre, 2008

Apéndice Lista de especies reconocidas del Maastrichtiense de la cuenca Neuquina-Colorado, modificado de Malumián et al. (1995); ${ }^{\circ}$ : especies que también se registran en el Daniense.

\section{Rotaliida}

Angulogavelinella? sp.

Anomalinoides pinguis (Jennings) = Anomalina pinguis Jennings, 1936.

A. sp., Bertels (1980).

“Anomalinoides" rubiginosus (Cushman) = Anomalina rubiginosa Cushman, 1926. Es una especie cosmopolita y euribatimétrica en la que Van Morkhoven et al. (1986) incluyen a Cibicides danica Brotzen, 1940 y Anomalina westraliensis Parr, 1938 como ecofenotipos ligados a diferentes profundidades. Aquí agregamos a Gavelinella jagueliana Bertels, 1980 como una forma de aguas muy someras. Anomalinoides rubiginosus es una especie bien establecida en la Patagonia, que se reconoce como Linaresia danica (Brotzen, 1940) en el Daniense de la cuenca del Golfo (Malumián \& Caramés, 1995), y en el Cretácico Superior y Paleoceno de cuenca Austral como Anomalina rubiginosa, (Natland et al., 1974). En la Antártida (Huber, 1988) se ha ilustrado como Anomalinoides rubiginosus y se ha descrito A. larseni Huber, 1988, que es muy próxima a G. jagueliana.

Charltonina acutimarginata $($ Finlay $)=$ Pulvinulinella acutimarginata Finlay, $1940=$ Charltonina kaasschieteri $($ Bertels $)=$ Alabamina kaasschieteri Bertels, 1980.

Cibicidoides sp. aff. C. succedens (Brotzen, 1948).

"Discorbis" correcta Carsey, 1926 = Discorbis correcta Carsey, 1926 = Planulina camachoi Bertels, 1964. Revets (2001) situó con dudas esta especie en el género Lingulogavelinella Malapris, 1965, pues su morfología no se condice con el de Gavelinella Brotzen, 1942, ni se ajusta a sus criterios de Lingulogavelinella, sin considerar la desconexión cronológica existente desde el Turoniense al Santoniense en la distribución de las especies del género, que también se verifica en la Patagonia. Se mantiene observada la asignación genérica original.

“D”. sp., Bertels (1980).

Gavelinella neuquense Bertels = Gavelinella? neuquense Bertels, 1980. Es una típica Gavelinella, de la que no se han encontrado formas similares.

Karreria pseudoconvexa $(\text { Parr })^{\circ}=$ Cibicides pseudoconvexus Parr, 1938. Ver sinonimia en Malumián \& Caramés (1995).

Nonionella cretacea Cushman, $1931^{\circ}$.

N. sp. cf. N. austiniana Cushman, 1933.

N.? sp., Bertels (1980).

"Paralabamina lunata (Brotzen)" = Eponides lunata Brotzen, 1948.

Patellina subcretacea Cushman \& Alexander, 1930.

Protelphidium primitivum Náñez \& Malumián n. sp.

Pullenia sp., Bertels (1980).

Rosalina patagonia Náñez \& Malumián n. sp.

Spirillina sp. cf. Turrispirillina subconica Tappan, 1943. 
Tehuelchella caminosi Náñez \& Malumián $n$. gen. n. sp.

Valvulineria? sp., Bertels (1980).

\section{Nodosariida}

Cytharina sp., Kielbowicz (1980).

C. sp.

Dentalina vertebralis (Batsch) = Nautilus vertebralis Batsch, 1791 .

Dentalina sp. aff. Nodosaria torsicostata ten Dam, 1944.

Dentalinoides sp. cf. Dentalina inornata d'Orbigny, 1846.

Frondicularia verneuiliana d'Orbigny, 1840.

"F." sp. 1.

Glandulina acuminata Costa, 1856.

Globulina gibba d'Orbigny, $1826^{\circ}$.

G. gravida $(\text { Terquem })^{\circ}=$ Guttulina gravida Terquem, 1878 .

G. inaequalis Reuss, $1850^{\circ}$.

Guttulina lactea $\left(\right.$ Walker \& Jacob) ${ }^{\circ}=$ Serpula lactea Walker \& Jacob, 1798.

G. lactea var. elongata Haynes, 1958.

G. problema d'Orbigny, $1826^{\circ}$.

G. problema var. arcuata Cushman, 1944.

Lagenoglandulina neuquensis $\left(\right.$ Bertels) ${ }^{\circ}=$ Pseudonodosaria neuquensis Bertels, 1980.

L. sp. cf. G. laevigata (d'Orbigny, 1826).

Lenticulina gryi $($ Brotzen $)=$ Astacolus gryi Brotzen, 1948.

L. navarroensis $($ Plummer $)=$ Cristellaria navarroensis Plummer, 1926

Pseudopolymorphina leopolitana (Reuss) = Polymorphina leopolitana Reuss, 1851 .

P. sp.

Pyramidulina marcki (Reuss) = Dentalina marcki Reuss, 1860 .

P. obscura (Reuss) = Nodosaria obscura Reuss, 1845 .

Ramulina globulifera Brady, 1879.

$R$. sp., Kielbowicz (1980).

Vaginulina? sp., Bertels (1978).

\section{Buliminida}

Baggatella coloradoensis Malumián, $1970^{\circ}$.

Bulimina kickapooensis Cole, 1938.

B. sp.

Buliminella isabelleana Camacho f. tuberculata Malumián \& Caramés, $1995^{\circ}$.

B. pseudoelegantissima Bertels, $1972^{\circ}$.

Cassidella tegulata (Reuss) = Virgulina tegulata Reuss, 1845.

Caucasina minuta Loeblich \& Tappan, $1964^{\circ}$

Coryphostoma decurrens (Ehrenberg) = Grammostomum(?) decurrens Ehrenberg, 1854 .

C. incrassata (Reuss) = Bolivina incrassata Reuss, 1851 .

C. plaita $($ Carsey) = Bolivina plaita Carsey, 1926.

C. sp., Bertels (1972).

Epistominella sp.

Fursenkoina sp., Bertels (1978).

Hiltermannella kochi (Bertels) = Hiltermannia kochi Bertels, 1970 .

Neobulimina argentinensis Bertels, 1972.

N. aspera Cushman \& Parker, 1940.

N. canadensis Cushman \& Wickenden, 1928.

Nodogenerina spinosa Hofker, 1956.

Praebulimina carseyi (Plummer) = Buliminella carseyae Plummer, 1931.

Praeglobobulimina jaguelensis Bertels, 1972.

Pseudouvigerina sp. aff. P. cimbrica Troelsen, 1945.

Pyramidina paleocenica $($ Brotzen $)=$ Bulimina paleocenica Brotzen, 1948.

P. prolixa $($ Cushman \& Parker $)=$ Bulimina prolixa Cushman \& Parker, 1935.

P. rugosa $($ Brotzen $)=$ Pseudouvigerina rugosa Brotzen, 1945 .

Tappanina? sp. = Tappanina sp. de Bertels (1980). Aparentemente los escasos ejemplares recuperados carecen de la sección rectangular y el diente apertural que caracteriza al género asignado, el que se mantiene con duda. Es posible que se trate de una nueva especie que encuentre mejor encasillamiento en el género Trachelinella Montanaro Gallitelli, 1956.

T.? sp., Bertels (1972).

Uvigerina elongata Cole, 1927. 


\section{Lituolida}

Dorothia bulletta $($ Carsey $)=$ Gaudryina bulletta Carsey, $1926^{\circ}$.

Gaudryina rudita Sandidge, $1932^{\circ}$. Tácitamente Malumián et al. (1995) consideraron a esta especie como un sinónimo joven de Gaudryina boltovskoyi Bertels, 1964. Por otro lado, existe una gran similitud entre los ejemplares de la Patagonia septentrional con los de Gaudryina healyi Finlay, 1939, de cuenca Austral.

Haplophragmoides sp., Bertels (1978).

Haplophragmoides sp.

Marssonella oxycona $($ Reuss $)=$ Gaudryina oxycona Reuss, 1860 .

Spiroplectammina laevis $\left(\right.$ Roemer ${ }^{\circ}=$ Textularia laevis Roemer, 1840.

Spiroplectammina spectabilis $\left(\right.$ Grzybowski) ${ }^{\circ}=$ Spiroplecta spectabilis Grzybowski, 1898.

Textulariella sp., Bertels (1978).

Tritaxia pyramidata Reuss, 1863

Trochammina ex gr. globigeriniformis (Parker \& Jones) = ex gr. Lituola nautiloidea var. globigeriniformis Parker \& Jones, 1865

\section{Uniloculares}

Favolagena atilai (Bertels) ${ }^{\circ}=$ Lagena atilai Bertels, 1964

F. ardolinoi Malumián, Náñez \& Caramés, 1991.

Fissurina carinata Reuss, 1963.

F. sp., Kielbowicz (1980).

Lagena acuticosta Reuss, $1862^{\circ}$.

L. acuticosta Reuss, 1862, sensu Bertels (1980).

L. hystrix Reuss, 1863.

Oolina sp., Malumián \& Náñez (1984).

\section{Miliolida}

Quinqueloculina sp., Bertels (1978).

Quinqueloculina sp. 1. 